Cipango Cahiers d'études japonaises

$18 \mid 2011$

Le Japon et le fait colonial I

\title{
Critiquer le colonialisme dans le Japon d'avant 1945
}

\section{Pierre-François Souyri}

\section{(2) OpenEdition}

\section{Journals}

\section{Édition électronique}

URL : https://journals.openedition.org/cipango/1525

DOI : 10.4000/cipango. 1525

ISSN : 2260-7706

\section{Éditeur}

INALCO

\section{Édition imprimée}

Date de publication : 1 juin 2011

Pagination : 189-236

ISBN : 978-2-85831-195-8

ISSN : $1164-5857$

\section{Référence électronique}

Pierre-François Souyri, «Critiquer le colonialisme dans le Japon d'avant 1945 », Cipango [En ligne], 18| 2011, mis en ligne le 18 juin 2013, consulté le 30 juin 2021. URL : http://journals.openedition.org/ cipango/1525; DOI : https://doi.org/10.4000/cipango.1525

Ce document a été généré automatiquement le 30 juin 2021.

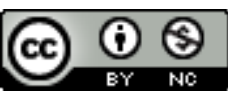

Cipango est mis à disposition selon les termes de la Licence Creative Commons Attribution - Pas d'Utilisation Commerciale 4.0 International. 


\title{
Critiquer le colonialisme dans le Japon d'avant 1945
}

\author{
Pierre-François Souyri
}

\author{
Sur la carte, on noircit la Corée à l'encre \\ Tandis que se fait entendre le vent d'automne. \\ 地困の上朝鮮国にくろぐろと \\ 墨をぬりつつ秋風をきく \\ Ishikawa Takuboku, 1910, \\ lors de l'annexion de la Corée par le Japon.
}

1 Dès les débuts de la mise en place des politiques coloniales, certaines voix se sont élevées au Japon pour critiquer cette nouvelle forme de domination sur des populations outre-mer. Avec les années dites de la démocratie Taishō (1912-1926), cette critique, peu connue, mais pourtant explicite du colonialisme, est devenue plus audible, avant d'être de nouveau progressivement étouffée à la fin des années 1930. À la différence des discours favorables à la liberté politique qui suscitent un vif écho dans le pays, la critique de l'impérialisme et du colonialisme ne fait cependant guère recette dans la société japonaise. Dans l'ancienne tradition du Mouvement pour la liberté et les droits $\mathrm{du}$ peuple des années 1880, les couches moyennes se reconnaissent dans les exhortations à plus de liberté et de démocratie et rejoignent en partie les aspirations ouvrières à l'amélioration des conditions de vie. Mais, dans sa grande majorité, la population pense qu'il y a - en plus de la fierté nationale - quelque chose à gagner dans les aventures expansionnistes qui débouchent sur la création d'un empire colonial.

2 Le mouvement indépendantiste en Corée, le boycott des produits japonais en Chine ou encore l'échec de l'entreprise militaire japonaise en Sibérie auraient pu inciter le Japon à plus de prudence en matière de politique extérieure et à entamer une autocritique de ses pratiques coloniales. Dans les cercles dirigeants japonais, on hésite en effet sur les méthodes de gouvernement. Latent depuis les premières expériences de colonisation à Taiwan à la fin du XIX ${ }^{\mathrm{e}}$ siècle, le débat éclate au grand jour en 1919. Les gouvernements coloniaux doivent-ils être laissés à l'armée ou placés sous administration civile? L'objectif de la colonisation est-il d'abord stratégique, (tenir les Occidentaux le plus 
possible à distance), économique (s'enrichir avec l'exploitation des colonies) ou bien at-il un objectif civilisateur avec l'extension des frontières japonaises en assimilant culturellement les peuples conquis ? Et d'ailleurs, ces peuples sont-ils assimilables ? Le débat s'engage sur le comment de la colonisation, voire sur le pourquoi, mais personne, dans les cercles dirigeants du moins, ne met en doute la colonisation en soi.

3 À Taiwan, la prise de contrôle de l'île à partir de 1895 s'était heurtée à une résistance qu'il faudra du temps pour éradiquer. Le gouvernement de la nouvelle colonie avait donc naturellement été placé entre les mains de militaires, ce qui d'ailleurs n'avait pas empêché des administrateurs civils comme Gotō Shinpei 後藤新平 (1857-1929) ou Nitobe Inazō 新渡戸稲造 (1862-1933) de travailler dans les années suivantes avec une certaine latitude, ce dernier étant plus particulièrement chargé sous les ordres du précédent du développement d'une politique agricole en milieu subtropical. De même, l'annexion de la Corée était intervenue, suite à l'assassinat du précédent résident général japonais et à des actions de guérilla menées contre le Japon, dans un contexte de forte tension. Les militaires de l'Armée avaient donc la main sur le système administratif colonial. Pour sa part, quand il forme le nouveau cabinet, le Premier ministre, Hara Takashi 原敬 (1918-1921) est hostile au contrôle des colonies par l'armée et partisan de leur en arracher le pouvoir en imposant une administration civile.

$4 \mathrm{Au}$ lendemain des manifestations antijaponaises qui enflamment la Corée au printemps 1919, les militaires, qui tiennent le pays et ont opté pour la répression brutale, sont critiqués à Tōkyō jusque dans les rangs du gouvernement. La réaction coréenne est interprétée comme une défiance contre le gouvernement militaire japonais en Corée plutôt qu'une véritable revendication nationale en faveur de l'indépendance. Après une phase de répression brutale, le régime colonial lâche du lest en abandonnant le régime de l'administration militaire (budan shugi 武断主義) et en prônant désormais une politique plus libérale dont l'objectif est le remplacement des militaires par des administrateurs civils censés moins brutaux et plus conciliants (bunji shugi 文治主義). Accueilli par les Coréens avec quelque soulagement, le gouvernement colonial, moins répressif que le précédent, se montre partisan de laisser se développer un discours culturel (bunka seiji 文化政治) coréen. On évoque partout au Japon l'unité de destin politique entre Japonais et Coréens (nissen dōji 日鮮同治). Cette politique est néanmoins vite soupçonnée dans la péninsule de n'être qu'une tentative pour briser la récente identité nationale des colonisés. Elle parvient surtout à diviser le mouvement national coréen qui hésite désormais entre nationalisme modéré «culturel» ou radicalisme indépendantiste. On constate "une instabilité désespérée », venant des tréfonds de la société coréenne face à la volonté assimilatrice du Japon ${ }^{1}$.

5 Très vite, des doutes naissent, au Japon même, sur la possibilité d'assimiler les peuples coloniaux. Rappelons que l'une des caractéristiques du colonialisme japonais est d'avoir toujours hésité entre un projet assimilationniste dōka seisaku 同化政策 (Taiwanais et Coréens deviendront un jour des Japonais à part entière) et un projet non assimilationniste dōka seisaku hantai ron 同化政策反対論 fondé sur l'idée d'une spécificité nationale nippone inaltérable et donc sur l'impossibilité des faire des colonisés des Japonais à part entière.

6 Les premiers sont animés d'une forme d'idéal qui assigne à la Nation japonaise un rôle civilisateur. Les colonisés sont des Japonais qui ne le savent pas encore. Il est de leur intérêt même de devenir japonais afin d'accéder aux bienfaits de la modernisation dans le cadre du Grand Japon. Cet idéal les encourage à accélérer, à la fin des années 1930, la 
transformation des colonisés en sujets du souverain (kōminka 皇民化), c'est-à-dire la "japonisation" des populations colonisées ${ }^{2}$. Des discours pseudoscientifiques sur l'origine commune des Japonais et des Coréens confortent volontiers leurs prétentions ${ }^{3}$. Certains iront même jusqu'à proposer à la fin des années 1930, des mariages mixtes entre les deux populations (hommes japonais et femmes coréennes) pour favoriser l'assimilation de la péninsule à la métropole (naisen ittai 内鮮一体). Cette politique aurait pour avantage de garantir définitivement la paix nippo-coréenne en créant un peuple métissé (kongō minzoku ron 混合民族論).

7 Souvent proches des cercles dirigeants de l'armée, les seconds sont colonialistes pour des raisons économiques et stratégiques, et leur orgueil national ne les pousse guère à imaginer que les populations colonisées soient assimilables, encore moins qu'elles puissent se métisser avec les peuples de la métropole. Mais du fait de leur inquiétude devant un éventuel soulèvement colonial qui risquerait d'affaiblir les positions extérieures de la zone de contrôle du Japon, ils sont enclins à se montrer partisans de laisser les colonisés gérer leurs affaires eux-mêmes, minzoku jiketsu seisaku 民族自決政 策. C'est pourquoi les assimilationnistes peuvent avoir parfois des positions politiques plus dures à l'égard des colonisés que ceux qui restent partisans de l'unicité de la 《race » japonaise et prompts à développer - c'est le cas de Tōgō Minoru 東郷実 - une théorisation de «la séparation des races » proche de l'apartheid (bunka seisaku 分化政 策 $)^{4}$. Les élites colonisées ont parfois joué de cette contradiction en soutenant alternativement plutôt l'un ou l'autre camp.

8 Rappelons par ailleurs que dès le milieu des années 1880, les partisans japonais de la liberté et des droits du peuple ont en tête l'idée que le Japon, parce qu'il est en train de se réformer et de se projeter en avant, doit aider les peuples alentour et tout spécialement la Corée à sortir de l'immobilisme. Gotō Shōjirō 後藤象二郎 (1838-1897) et Itagaki Taisuke 板坦退助 (1837-1919), les dirigeants du Parti de la Liberté (Jiyūtō 自 由党), ont le projet en 1884 d'un coup d'État militaire en Corée pour débarrasser la péninsule du traditionalisme de la monarchie en place dans la péninsule et qui constitue un obstacle à la pénétration de la civilisation ${ }^{5}$. Gotō rêvait même de devenir le Premier ministre d'une Corée libérée! L'année suivante, ōi Kentarō 大井憲太郎 (1843-1922), le leader de l'aile la plus radicale du Parti de la Liberté envisage à son tour de prendre les armes pour libérer la Corée de l'emprise conservatrice et renverser le gouvernement avec des moyens militaires ${ }^{6}$. L'intellectuel Tsurumi Shunsuke (1922-) fait remarquer qu'à la fin du $\mathrm{xIx}^{\mathrm{e}}$ siècle, les courants politiques « de gauche comme de droite » sont tous en accord pour imposer la civilisation, par la force si nécessaire ${ }^{7}$. En 1905, le journaliste et historien Takekoshi Yosaburō 竹越与三郎 (1865-1950) écrit dans une étude sur le gouvernement colonial à Taiwan dix ans après l'annexion :

On croyait que c'était le fardeau de l'homme blanc pour l'éternité que de défricher des territoires vierges et d'y importer les vertus de la civilisation. Mais maintenant la nation japonaise s'est éveillée sur les rivages de l'Orient extrême et veut partager ce fardeau avec les Blancs. Serions-nous aussi en charge, sans le savoir, de porter le fardeau de l'homme jaune? De la réussite ou de l'échec de l'expérience taiwanaise dépendra la réponse ${ }^{8}$.

De son côté, le socialiste (et futur anarchiste) Kōtoku Shūsui 幸徳秋水 (1871-1911) avait entamé, en 1901, une critique de l'impérialisme qu'il considérait surtout comme un fauteur de guerre favorisant les tendances militaristes et despotiques. Toutefois, il ne s'était guère penché sur la question coloniale, et n'avait pas fait de lien entre l'impérialisme et l'exploitation coloniale en tant que telle ${ }^{9}$. Ce n'est donc que peu à peu, 
au début des années 1910, qu'émergent les premiers courants critiques. Puis, sous l'impact de la révolution russe, du droit à l'autodétermination, de l'agitation sociale qui traverse le pays, de la progression des idéaux démocratiques, que certains commencent à s'en prendre directement à l'impérialisme et à sa conséquence immédiate et sensible, le colonialisme. Les courants socialistes, communistes et anarchistes manifesteront toujours une méfiance instinctive devant le colonialisme. Dans leur esprit, celui-ci reste en effet associé à l'impérialisme et au bellicisme, comme l'avait soutenu Kōtoku. Pourtant, la solidarité avec les peuples colonisés paraît secondaire dans leur combat, et rares sont ceux qui font de la lutte anti coloniale l'une de leurs priorités. De leur côté, certains libéraux partent d'une analyse fondée sur les coûts économiques pour montrer que les colonies font dépenser au pays plus qu'elles ne lui rapportent. Immorales, brutales et en définitive irresponsables pour les uns, les politiques coloniales sont surtout dispendieuses et inefficaces pour les autres. Entre ces deux positions défendues comme on le verra par des personnalités éminentes, on retrouve tout un arc de positions intermédiaires, dans lesquelles l'indignation se mêle parfois au calcul économique. D'autres enfin s'offusquent de l'ignorance des administrations coloniales vis-à-vis des cultures locales et critiquent leur volonté de les nier ou de les détruire au nom d'un projet colonial présenté comme moderne et civilisé.

On peut distinguer trois courants principaux qui s'en prennent aux politiques coloniales du Japon.

11 D'abord un courant «moral» qui émerge essentiellement comme critique des méthodes répressives policières et militaires dans les colonies, surtout au lendemain des mouvements de 1919. Les porte-parole de ce courant s'indignent au nom d'une politique dont on pense qu'elle n'a de sens que pour accomplir le «bien». Or elle produit du «mal». Ils prennent position en faveur des "autres", "pensent aux Coréens ", mais ne sont pas nécessairement hostiles au projet colonial en tant que tel. L'entreprise leur parait en soi positive, mais doit être réformée, améliorée. En fin de compte, elle devrait permettre de "civiliser", de "moderniser", voire de "démocratiser" les sociétés colonisées. La plupart des tenants de ce courant se montrent assez virulents contre les politiques d'assimilation et évoluent vers des positions de plus en plus critiques face à la brutalité inhérente du système. Sont soulignées l'originalité et l'importance des cultures des peuples colonisés ou semi colonisés, et est affichée une claire hostilité au rouleau compresseur de la "politique culturelle " pratiquée par le Japon colonial. Ce courant - encore marginal avant 1919 s'exprime de plus en plus nettement dans les années vingt et trente.

Il existe par ailleurs un courant «économiste» produit de la pensée libérale occidentale. Ce courant, finalement assez radical, est tout sauf altruiste. Il émerge au début des années Taishō et dénonce les politiques coloniales et plus largement impérialistes comme non profitables, inutiles et dangereuses, car conduisant à terme à la guerre. Ces politiques nécessitent une augmentation des budgets militaires jugés improductifs. Colonialisme et impérialisme sont contraires aux intérêts bien compris du pays, de l'État, de la Nation - et qui plus est, sont moralement peu recommandables. Ce courant est anticolonial par principe, mais se préoccupe peu des mouvements des peuples colonisés, sinon pour proclamer qu'ils conduisent inéluctablement à l'indépendance et donc à la défaite programmée du Japon.

Enfin, il apparaît une critique du système au sein même de l'université japonaise parmi certains professeurs chargés d'enseigner précisément les politiques coloniales. 
Yanaihara Tadao 矢内原忠雄 (1893-1961), en poste à partir de 1923, est de ce point de vue emblématique. D'une certaine façon il tente une synthèse entre les deux courants résumés ici, alliant une critique économique, une critique morale et la prise en compte des aspirations des colonisés. Dans les années 1930, il commence à entrevoir l'indépendance des colonies comme la finalité à terme du processus engagé.

Chacun de ces courants rapidement définis ici s'incarne à un moment ou à un autre dans des «figures » dont nous évoquerons ici le parcours et les idées politiques. Bien entendu, ces penseurs ont pu s'influencer réciproquement et certains critiques du colonialisme ont pu, selon l'époque ou selon leur cheminement politique propre, porter l'accent de leur critique tour à tour sur l'un ou l'autre de ces aspects : le colonialisme est moralement inacceptable parce que fondamentalement brutal et oppressif, culturellement stupide parce qu'ignorant et destructeur des réalités locales, économiquement coûteux parce que non rentable pour la Nation.

\section{Dénoncer la répression, promouvoir la culture des colonisés}

Les mouvements indépendantistes ou nationalistes qui éclatent au printemps 1919 en Corée et en Chine sont rapportés au Japon dans les principaux médias comme des mouvements antijaponais pour l'essentiel et, de ce fait, ne suscitent guère de sympathies dans l'archipel. Selon certains journaux japonais, les Coréens auraient même été manipulés par des missionnaires chrétiens occidentaux hostiles à la présence japonaise dans la péninsule.

16 L'un des premiers à dénoncer cette analyse simpliste des mouvements sur le continent est un professeur de sciences politiques à l'Université Impériale de Tōkyō, Yoshino Sakuzō 吉野作造 (1878-1933). Depuis 1905, Yoshino se montre partisan de la doctrine $\mathrm{du}$ «régime constitutionnel à l'intérieur, impérialisme à l'extérieur» (uchi ni rikkenshugi, soto ni teikokushugi 内に立憲 主義、外に帝国主義), mais ses séjours en Chine et en Corée puis sa réflexion politique personnelle le poussent à prendre peu à peu ses distances avec les positions qu'il défendait dans sa jeunesse. Le virage politique est pris entre 1916 et 1918 et repose sur une certaine confiance dans la montée des forces démocratiques au Japon, mais aussi en Chine et en Corée. Yoshino parle en faveur d'une démocratie qui serait compatible avec la Constitution de 1889 et qui pourrait se développer dans le cadre du système impérial. Il la nomme minpon shugi 民 本主義, la démocratie impériale ${ }^{10}$. Désormais, l'idéal qu'il proclame, c'est «la réalisation de la démocratie à l'intérieur et de l'égalité entre les peuples au niveau international » (uchi ni minpon shugi no tettei, soto ni kokusai byōdōshugi no kakuritsu 内に 民本主義の徹底、外に国際平等主義の確立), et il se montre en faveur du droit à l'autodétermination des peuples (minzoku jiketsu 民族自決) ${ }^{11}$.

17 Comme beaucoup d'observateurs politiques japonais, Yoshino Sakuzō a été très impressionné par la révolution chinoise nationaliste de 1911, en proclamant la république. Il se sent proche des révolutionnaires chinois ${ }^{12}$. Il rédige d'ailleurs une histoire de la révolution chinoise qu'il publie en $1917^{13}$. Dans des articles publiés dans les années suivantes, il commence à critiquer la manière brutale avec laquelle agit le gouvernement japonais sur le continent et prône un rapprochement entre le Japon et les jeunes nationalistes chinois qui «ont entre leurs mains l'avenir du pays ${ }^{14}$. Plus 
encore, il critique de plus en plus violemment les méthodes de la kenpeitai 憲兵隊, la gendarmerie japonaise opérant dans les territoires extérieurs ${ }^{15}$, et émet à regret de sérieux doutes sur les possibilités ultimes d'assimiler le peuple coréen à la nation japonaise, faisant valoir que la péninsule coréenne possède depuis longtemps une civilisation à part entière, différente de celle de l'archipel japonais.

18 Le professeur accueille avec joie des étudiants chinois et coréens dans ses cours et séminaires, et les invite chez lui à discuter librement, ce qui, dans le contexte, ne va pas toujours de soi. Il voyage de nouveau en 1916 en Chine et en Corée et avec ce séjour, les premiers doutes apparaissent :

J'ai rencontré cet an-ci beaucoup de Coréens et quand on écoute ce qu'ils disent, on voit qu'ils sont, contre toute attente, nombreux à évoquer l'injustice actuelle des autorités politiques japonaises dans leur pays. Que cette injustice soit réelle ou non, peu importe, mais nous aurions tort de négliger ce qu' ils racontent ${ }^{16}$.

19 À la fin de la Première Guerre mondiale, la poussée nationaliste et sociale en Chine et en Corée conduit Yoshino à prendre encore plus franchement ses distances vis-à-vis de la politique du Japon. En octobre 1918, il rédige un court article dans lequel il cite les propos du professeur d'études coloniales de l'Université de Kyōto, Yamamoto Miono 山 本美越乃 (1874-1941) qui, dans le quotidien Ōsaka Mainichi shinbun 大阪毎日新聞, expliquait que les Coréens «possédaient leur propre culture depuis toujours » et qu'il était «vain d'ignorer leur habitudes, leurs institutions et leur mœurs pour les gouverner ». Et en conclusion, Yoshino se fait prophétique :

La question coréenne va devenir, dans un avenir proche, le principal souci politique

du Japon: nous devons réaliser qu'à la faveur de la guerre, les courants

nationalistes en Corée sont devenus très importants ${ }^{17}$.

20 À la suite du mouvement nationaliste coréen de mars 1919 au cours duquel les manifestations antijaponaises se multiplient ${ }^{18}$, Yoshino Sakuzō décide d'inviter ses étudiants coréens aux réunions de la Reimeikai ${ }^{19}$ et leur donne la parole pour qu'ils s'expriment en faveur de l'indépendance de leur pays, sous les applaudissements du public. Il explique qu'il faut aussi savoir se placer du point de vue des Coréens pour comprendre ce qui se passe. Une partie - minoritaire, mais conséquente - de l'opinion le suit. Dans l'une de ces réunions, le professeur Fukuda Tokuzō 福田徳三 (1874-1930) déclare tout de go que « la Corée n'est pas la propriété des cliques militaristes » et qu'il « est grand temps d'y appliquer la loi constitutionnelle $»^{20}$. Mais les articles de Yoshino suscitent aussi l'hostilité grandissante de l'opinion nationaliste.

21 Quelques semaines plus tard, à l'occasion du mouvement chinois du 4 Mai 1919, Yoshino Sakuzō est l'un des rares intellectuels à intervenir dans la presse japonaise en faveur des nationalistes chinois qui boycottent les produits japonais. Il explique que ces mouvements « qui ont rendu quelque peu les Japonais nerveux », visent la bureaucratie de Tōkyō, les cliques militaires et financières et non le peuple japonais en tant que tel.

Depuis de longues années, je lutte pour libérer mon pays bien aimé des mains des cliques bureaucratiques et militaires. Je n'ai pas l'impression que les étudiants de Pékin soient en ce moment en train de faire autre chose. Je ne peux que souhaiter le succès d'un pareil mouvement ${ }^{21}$.

Yoshino parvient, dans la foulée, à inviter à Tōkyō une délégation de professeurs et d'étudiants de l'Université de Pékin "pour que les jeunesses des deux pays se comprennent mieux». D'ailleurs, vers la même époque, un grand nombre de professeurs et de journalistes démocrates se déplacent en Mandchourie, à Pékin ou à Shanghai pour tenter de comprendre ce qui se passe sur le continent. En mai 1920, les 
invités chinois interviennent publiquement pour expliquer les contenus du Mouvement du 4 Mai devant les membres de la Reimeikai ou devant la Shinjinkai ${ }^{22}$. Les étudiants les accueillent avec attention et les questionnent longuement. Il en va de même de l'intérêt de ces associations pour une réflexion sur les problèmes coloniaux, et notamment sur la Corée. On se prononce en faveur d'une «amélioration» (kaizen 改善) de la politique menée dans la péninsule par le Japon ${ }^{23}$. On notera que les numéros de la revue de la Shinjinkai consacrés à la Corée sont frappés par la censure et interdits de publication. Yoshino dénonce la politique de répression menée par le gouvernement colonial japonais en Corée en 1919 (8000 morts et 45000 arrestations) ${ }^{24}$ qu'il qualifie d'« inadmissible » et d'《immorale $»^{25}$ et notamment les massacres perpétrés à Suwon en 1919 et dans le district de Jiandao 間島 (dans la zone de la Mandchourie où réside une forte population coréenne ${ }^{26}$ en octobre 1920 par les forces de l'ordre japonaises, dont ni le gouvernement ni même la presse n'avaient rendu compte ${ }^{27}$. Puis dans des articles favorables aux revendications du mouvement coréen, il exige comme réponse aux manifestants la suppression des pratiques discriminatoires du gouvernement colonial à l'encontre des Coréens, la fin du régime militaire dans la péninsule, l'abandon de la politique d'assimilation et la liberté d'expression.

Malgré ses prises de position courageuses et sa sympathie pour les mouvements nationalistes chinois et coréens qu'il considère comme des mouvements d'aspiration démocratique, Yoshino Sakuzō ne verse jamais dans l'anticolonialisme. Il s'exprime en faveur d'un colonialisme libéral utopiste qui ne réprimerait pas les mouvements indépendantistes et laisserait plus de liberté et de démocratie aux populations colonisées, mais ne remet jamais en question le principe de la colonie.

Nul doute que les Coréens sont sur le plan juridique des sujets japonais. Mais dans la réalité les Coréens ne sont pas de la race du Yamato. Dans l'Empire du Grand Japon qu'est en train de construite le peuple du Yamato, les Coréens occupent comme une place de lointain rejeton et cela en vérité est bien difficile à cacher. Nous aimerions pouvoir espérer avec enthousiasme que les Coréens éprouvent vis-à-vis du Japon les mêmes sentiments de loyauté que les habitants de la métropole, mais il va de soi que l'on ne peut leur imposer de force un pareil sentiment et même si c'est dur à dire, cela n'est pas d'actualité28.

Face au mouvement nationaliste coréen de mars 1919 et à sa répression de la part des autorités japonaises dans la péninsule, Yanagi Sōetsu (1889-1961) lui aussi réagit fortement contre l'attitude du gouvernement de Tōkyō et se fait l'avocat des Coréens. "Constatant que personne ne prenait la défense publique des malheureux Coréens, j'ai écrit en vitesse ${ }^{29}$, dira-t-il à propos de "Penser aux Coréens » texte percutant qu'il a publié dans le quotidien Yomiuri shinbun (20-22 mai 1919). "Qui peut croire vraiment qu'on peut lier les gens entre eux avec un gouvernement militaire et de la répression ?... L'amoureux de la paix ne peut ici que dessiner sur ses lèvres un sourire amer $»^{30}$. Il ajoute :

Si nous recherchons la paix éternelle avec nos voisins, il n'y a d'autre voie que de purifier notre cœur dans l'amour et de baigner dans la compassion. Or malheureusement, le Japon a sorti le sabre et manie l'insulte. Est-ce ainsi que l'on fait naître une compréhension mutuelle, une coopération, une association? Non. Ce que ressentent les Coréens, c'est une indignation sans limites, une volonté de résistance, de la haine, un désir de séparation. Le résultat de tout ceci, c'est qu'ils n'ont plus qu'un seul idéal, l'indépendance. Il est normal qu'ils n'aiment pas le Japon et ceux qui portent de l'estime à notre pays ne sont qu'une poignée ${ }^{31}$. 
aujourd'hui comme le fondateur du mouvement pour la reconnaissance d'un artisanat populaire, critique d'art et philosophe des religions, Yanagi Sōetsu ${ }^{32}$ se dresse pourtant contre la pensée dominante de son temps, autrefois incarnée par Fukuzawa Yukichi 福沢諭吉 (1835-1901) dans les années 1880 dans son essai "Adieu l'Asie » (Datsu A ron 脱亜論), ou encore contre celle formulée un peu plus tard dans les années 1890 par les tenants du nationalisme projaponais (kokusuishugi 国粋主義). Cette manière de penser conduisait, selon Yanagi Sōetsu, à développer un sentiment d'infériorité vis-à-vis des Occidentaux et un complexe de supériorité vis-à-vis des autres Asiatiques. À la différence de bien de ses contemporains, Yanagi ne pensait pas que les Japonais étaient "supérieurs » aux Coréens, ce qui, en son temps, était bien rare $^{33}$. Yanagi Sōetsu, lui, ne développe en tout cas ni un sentiment d'infériorité vis-àvis des Occidentaux, ni ne ressentait leur existence comme une pression. Sa pensée se construit dans ce qu'il faut bien appeler un cadre favorable, la période démocratique de l'ère Taishō. Nombreux sont ceux qui, à cette époque, pensent que le Japon, devenu enfin l'égal des grandes puissances, allait être considéré sur un pied d'égalité par les autres pays occidentaux, et ainsi s'arracher à ce désagréable sentiment d'infériorité développé par certains Japonais à l'égard des « Blancs », le sentiment dit gaison naihi 外 尊内卑 (Aduler l'étranger et se dénigrer soi-même), évoqué déjà dans les années 1890 par le nationaliste Miyake Setsurei 三宅雪嶺 (1860-1945).

Issu de Gakushūin 学習院, l'école de l'aristocratie, membre des plus influents du groupe littéraire Shirakaba 白樺, Yanagi Sōetsu se lie très tôt avec des Occidentaux qui séjournent au Japon. En particulier, il se noue d'amitié avec Bernard Leach, grand connaisseur de la culture artistique de l'Extrême-Orient, futur auteur du Livre du Potier et introducteur en Europe de la céramique japonaise raku ${ }^{34}$. Avec Leach, Yanagi voyage en Corée à plusieurs reprises et Leach initie Yanagi à la beauté des céramiques coréennes. La fascination de Leach pour l'art d'Extrême-Orient n'a d'égale que celle de Yanagi pour les arts populaires japonais, coréens et même chinois. Yanagi est en particulier émerveillé en 1914 par la beauté des céramiques blanches de l'époque de la dynastie Yi, des céramiques jugées généralement comme "ordinaires » que personne, avant lui, n'avait véritablement appréciées. Il est l'un des premiers Japonais à considérer sans préjugé la culture coréenne et en apprécier l'esthétique, « une beauté amère ${ }^{35}$ rendant ainsi hommage à ceux qui ont créé ces œuvres. Il se voit un peu comme Lafcadio Hearn qui fit tant pour mieux faire connaître la culture japonaise en Occident $^{36}$. Pour les Japonais qui ne considéraient jusqu'alors la Corée que comme un pauvre pays misérable, le discours de Yanagi est vraiment novateur. Il fait découvrir à ceux qui l'écoutent " une autre Corée ». La beauté transcende les frontières, explique Yanagi qui se rend à de nombreuses reprises dans la péninsule et commence à collectionner des pièces d'artisanat local.

Mais Yanagi n'est pas qu'un commentateur de la vie politique et il ne se borne pas à adopter une attitude purement esthétisante. Son discours n'est pas seulement une critique de la violence répressive. C'est aussi un plaidoyer pour la défense culturelle de la Corée et il s'en prend aux pratiques et aux modes de raisonnement des Japonais.

Nous utilisons des méthodes qui ont d'abord pour objectif de rendre impossible l'indépendance totale des Coréens. Nous ne reconnaissons pas non plus qu'ils puissent avoir leurs propres manières de penser et nous ne leur offrons qu'un enseignement ou une morale adaptés au Japon. Bref, qu'il s'agisse des choses matérielles ou spirituelles, nous leur avons volé leur liberté et leur indépendance. Certains disent que c'est ainsi que nous semons les graines qui leur permettront de 
penser à la japonaise, mais nous n'essayons jamais d'émouvoir leur cœur. Quand nous nous approchons d'eux, c'est toujours avec le sabre, jamais avec le cœur ${ }^{37}$. géographiquement, ethniquement, linguistiquement proches comme des frères. La situation actuelle n'est absolument pas normale... La Corée est comme un frère pour le Japon et celui-ci la traite en esclave $»^{41}$. En s'adressant directement aux Coréens, en évoquant ses nombreux amis coréens connus et inconnus, Yanagi utilise un procédé rhétorique efficace qui permet à son lecteur japonais de se déplacer dans la peau du colonisé. Dans un texte de 1920, il écrit que pour les Coréens, le Japon n'est sans aucun doute rien d'autre qu'un pays violent et impitoyable ${ }^{42}$. Il donne à voir sans fard le point de vue de l'autre avec lequel il entre en empathie pour dénoncer le système. Il demande parfois à son lecteur de se mettre dans la position inverse: "Ah si les Japonais pouvaient se mettre à la place des Coréens... » écrit-il à plusieurs reprises ${ }^{43}$. Il critique aussi la stupidité du système éducatif mis en place en Corée par les autorités coloniales, qui vise à implanter les valeurs japonaises en niant l'histoire de la culture coréenne. Très hostile à la politique d'assimilation, il fait dire à un Coréen : « le Japon nous offre une éducation... c'est pour nous ou c'est pour lui ? On demande aux jeunes Coréens de considérer comme des héros ceux qui ne sont que des voleurs $»^{44}$.

31 Pour Yanagi, les Japonais doivent faire preuve sur ces questions d'un sens minimum de l'autocritique. "J'aimerais faire savoir aux Coréens qu'il y a, parmi nous autres Japonais, des gens qui pensent clairement que, dans cette affaire, le Japon foule la morale aux pieds $»^{45}$. Et dans son texte adressé à des amis coréens, il critique «le Japon de la force » auquel il préfère un Japon plus compassionnel, et souhaite des «Japonais humains ». Il considère la situation actuelle en Corée " anormale », " non naturelle ». 
Une partie de son texte est censurée par les autorités et sa traduction dans un journal de langue coréenne est suspendue ${ }^{46}$.

Quand le gouvernement colonial indique vouloir détruire la Porte Kwanghwa du palais Kyŏngbok, à Séoul, pour édifier une énorme bâtisse moderne destinée à devenir le nouveau siège du gouvernement général, Yanagi est scandalisé. Si les colonisateurs y voient symboliquement une victoire de la modernité japonaise sur le passé coréen (la porte originale avait été édifiée en 1395), les Coréens y voient une atteinte à un symbole de leur histoire nationale. Yanagi juge que les autorités japonaises commettent là une bêtise supplémentaire, celle de bureaucrates incultes et aveugles qui ignorent tout du passé de la péninsule et du sentiment national naissant. Il publie un article dans Kaizō, en septembre 1922, sur les trésors architecturaux coréens en passe d'être détruits par les Japonais, et son article prend la forme d'une lettre qu'il adresse à la Porte Kwanghwa: "Porte Kwanghwa, Porte Kwanghwa, tes jours sont désormais comptés. Tous les souvenirs qui te sont associés en ce monde sont donc voués à disparaître dans un froid oubli $»^{47}$. Il dresse l'éloge de ce bâtiment: «Ô chère Porte Kwanghwa de si admirable apparence $»^{48}$ qui possède « beauté tranquille et dignité ». Yanagi manifeste ainsi sa sympathie au peuple coréen que l'on va priver d'une part de lui-même, défend l'art en général et conteste l'idée saugrenue de construire une bâtisse moderne "totalement dépourvue de beauté créative » dans un pareil lieu chargé d'histoire. Il regrette que personne ne puisse prendre vraiment la défense du monument et, pire encore, que ceux qui s'opposent à sa destruction soient accusés d'être des comploteurs ${ }^{49}$. Il n'hésite pas à prendre à partie son lecteur japonais : «Lecteur, il ne faut pas mépriser cette porte en déclarant qu'elle n'est qu'une œuvre de la fin de la période des Yi $»^{50}$. Et de critiquer une politique qui se comporte vis-à-vis de l'art sans aucune retenue. « Ô Porte Kwanghwa, comme tu dois être triste! » Par sa véhémence et son ironie teintée de désespoir, il touche une partie des décideurs japonais qui se résolvent à construire leur bâtiment, mais sans détruire ladite Porte qui, démontée, sera finalement reconstruite en un autre lieu ${ }^{51}$. Yanagi est alors fiché par les autorités japonaises comme « personne dangereuse $»^{52}$.

La «découverte» par Yanagi Sōetsu d'un «art populaire » issu de l'artisanat traditionnel en Corée, au moment où l'État japonais réprime les populations de la péninsule, le pousse à prendre des positions qui frisent l'anticolonialisme. Que l'indépendance soit devenue pour les Coréens un idéal, voilà qui n'est que la nécessaire conséquence du ressentiment qu'ils éprouvent pour leurs oppresseurs, pense Yanagi. Mais son impuissance à peser sur les décisions politiques prises à Tōkyō ou à Séoul par les autorités coloniales le démoralise. Avec certains de ses amis japonais de Séoul et acquis à la cause coréenne (il en existait quelques-uns comme les frères Asakawa Noritaka et Takumi ${ }^{53}$, qui avaient décidé de s'établir sur place, au début des années 1910, par amour pour l'art et l'artisanat de Corée, et qui aidaient Yanagi à comprendre l'art coréen), il entretient des relations d'amitié avec des intellectuels et des artistes coréens comme Yom Sangso ou Nam Kyŏngbok, des nationalistes réformistes qui jouèrent l'un et l'autre un rôle important en Corée dans le travail de mise en place d'une histoire de la littérature coréenne et d'une histoire des mouvements du peuple coréen. En 1924, Yanagi fonde à Séoul un petit Musée des Arts populaires coréens. Il déclare avoir pour ambition d'aider les Coréens eux-mêmes à mieux comprendre et apprécier leur propre culture. 

promoteur d'une forme de légitimité coloniale. Par son action de promotion de la culture coréenne, Yanagi aurait finalement participé à la stabilisation du régime japonais en Corée en devenant comme la vitrine du projet japonais de "politique culturelle». Nommé par le Premier ministre Hara Takashi en 1919, le nouveau gouverneur général, l'amiral Saitō Makoto 斎藤実 (1858-1936), passait pour un libéral. Saitō, d'ailleurs, fournit une aide aux projets de Yanagi car il était l'un des anciens subordonnés du père de Yanagi Sōetsu. Ceci expliquerait en partie les facilités dont ce dernier aurait bénéficié de la part de certains représentants de l'autorité coloniale quand il cherche à monter un musée ${ }^{54}$. Il est certain que le point de vue discutable de Yanagi sur la «tristesse» de l'esthétique des pièces coréennes a le don d'hérisser aujourd'hui certaines sensibilités en Corée. Mais il faut quand même contextualiser le discours de Yanagi et, même si l'homme n'était pas exempt de défauts, il n'en a pas moins contribué à faire connaître une céramique largement mésestimée. Après tout, où sont les esthètes français capables dans les années 1920 de monter en Algérie un musée des arts populaires algériens?

À la suite du Grand tremblement de terre du Kantō, des pogroms éclatent en septembre 1923, principalement dirigés contre les immigrés coréens dont plusieurs milliers sont massacrés, sans que la police ne juge bon d'intervenir. Aussitôt Yoshino Sakuzō prend sa plume pour dénoncer les massacres et il va même jusqu'à démontrer que la police a souvent armé le bras des assassins. Ses articles finissent par émouvoir une partie de l'opinion et même des membres du cabinet qui exigent l'arrêt des massacres ${ }^{55}$. La revue de la Shinjinkai publie un numéro spécial un mois après le séisme, "Tanemaku hito no tachiba» 種莳く人の立場 (Dans la position du semeur), qui évoque ces violences « avec répugnance ». " On a beau essayer et essayer encore, on ne peut effacer cela $»^{56}$.

L'année suivante, l'hystérie gagne les médias japonais au moment où la Chambre des Représentants américaine s'apprête à faire passer une nouvelle loi sur l'immigration (Immigration Act) qui comporte des clauses ouvertement racistes et antijaponaises. Yamakawa Kikue 山川菊栄 (1890-1980), une féministe proche des communistes, dénonce en mai 1924 le mouvement antijaponais aux États-Unis et critique au nom de l'égalité des races l'égoïsme des Américains, mais elle continue :

Lors du grand séisme et du grand incendie de San Francisco [en 1907], la police et l'armée américaines ont-elles pris prétexte de la catastrophe pour manifester clairement leurs sentiments antijaponais et leurs préjugés de race ? Ont-elles essayé de massacrer en grand nombre des Japonais? Des Japonais, aux États-Unis, ont-ils connu le même destin que les Coréens et certains travailleurs lors du grand tremblement de terre de l'automne dernier [septembre 1923]. Les Coréens, les Taiwanais et les gens issus d'autres peuples vivant au Japon ont-ils vraiment un traitement identique à celui des Japonais de métropole sur le plan politique, social ou économique ? Et parmi les Japonais de métropole, les femmes et les travailleurs ont-ils vraiment les mêmes droits que les autres, alors qu'ils ont - et c'est normal les mêmes devoirs? Et même pour les Japonais, il existe au Japon beaucoup trop de zones sanctuarisées où, en fonction du sexe, du degré d'éducation ou de la fortune, certains lieux sont interdits d'accès comme si des panneaux à l'entrée indiquaient : «ici on n'entre pas si on est issu de catégories ou de peuples inférieurs »"

Ce texte qui prend prétexte de l'attitude jugée inacceptable des autorités américaines, est parmi les premiers non seulement à dénoncer le statut et la condition des peuples colonisés au sein même de la métropole coloniale japonaise, mais aussi à évoquer la nécessité d'extirper dans un seul mouvement la discrimination générale dont sont

Cipango, 18 | 2011 
frappés certains groupes au sein de la société. Yamakawa Kikue reconnait la nécessité de lier le combat des peuples coloniaux au mouvement ouvrier et à celui des femmes ${ }^{58}$.

\section{Grand ou Petit Japon?}

Parmi les intellectuels démocrates, Ishibashi Tanzan 石橋湛山 (1884-1973) est de son côté connu pour sa dénonciation du phénomène impérialiste. Partisan d'un «Petit Japon» (shō nippon shugi 小日本主義), Ishibashi pense, pour des raisons qu'il veut rationnelles, que l'impérialisme et le colonialisme coûtent plus cher sur le long terme qu'ils ne rapportent et qu'ils favorisent la présence inopportune et envahissante de cercles militaristes dans les organes centraux de l'État. Ishibashi Tanzan est l'un des premiers à penser que l'expansionnisme travaille contre la démocratie. En ce sens, il tranche avec un Yoshino Sakuzō qui, longtemps, appuya la thèse de "la démocratie à l'intérieur et de l'impérialisme à l'extérieur ». En raison même de son destin aprèsguerre (il devient ministre des Finances en 1946-47, puis Premier ministre quelques semaines en 1956-57), les positions d'Ishibashi Tanzan ne sont évidemment pas anecdotiques ${ }^{59}$.

Ishibashi Tanzan est issu de l'École spéciale de Tōkyō (Tōkyō senmon gakkō 東京専門 学校, la future Université de Waseda). Philosophe de formation, il a été influencé par la pensée pragmatique de John Dewey (il invente en japonais une traduction du mot pragmatisme : sayōshugi 作用主義). Il entre à l'agence Tōyō qui édite plusieurs organes de presse. Il écrit d'abord dans Tōyō jiron 東洋時論, un magazine fondé en 1910 qui se dresse contre l'ancienne morale au nom de l'individualisme, soutient le tout nouveau mouvement des femmes et critique la répression du gouvernement impérial contre le socialisme. Au moment de "l'affaire du crime de lèse-majesté » en 1911, Tōyō jiron, révolté par les condamnations à mort prononcées contre les anarchistes, est interdit par la censure à plusieurs reprises. Peu après, Ishibashi entre - sans réelle formation économique - à la revue Tōyō keizai shinpō 東洋経済新報 (Nouvelles économiques de l'Orient). En plus des affaires économiques et financières, cette revue traite également, d'un point de vue libéral, les problèmes politiques, diplomatiques et sociaux. Elle est notamment hostile à l'extension permanente des budgets militaires. Fief des anciens de Waseda, Tōyō keizai shinpō est considéré à l'époque comme l'un des bastions du libéralisme radical (kyūshinteki jiyūshugi 急進的自由主義), et devient vite dans les années qui suivent une revue de référence pour ses prises de position souvent lucides et anticonformistes. Le groupe est dirigé par Miura Tetsutarō 三浦鉄太郎, le mentor d'Ishibashi. Ce dernier fait une carrière brillante dans cette revue dont il devient l'une des plumes les plus remarquables, puis finalement le directeur. Sa formation économique est celle d'un autodidacte. Ishibashi Tanzan devient un vrai libéral, disciple d'Adam Smith, et parle aussi en faveur d'accords de libre-échange entre l'archipel et la péninsule ${ }^{60}$. Malgré son libéralisme sans faille à l'heure de l'interventionnisme étatique tous azimuts, Ishibashi sera souvent consulté dans les années 1930 par les cercles dirigeants pour ses expertises économiques ${ }^{61}$.

Dès le milieu des années 1910, Ishibashi Tanzan concentre sa critique sur la politique extérieure de Tōkyō, sur «la doctrine du Grand Japon », (Dai Nipponshugi 大日本主義) qui a pour conséquence de vouloir produire toujours plus d'armements. Il faudrait au contraire se focaliser sur une vision différente, celle d'un « Petit Japon », explique-t-il. L'idée lui en a été glissée en fait par Miura Tetsutarō qui, en 1913, signe un article 
intitulé « Grand ou Petit Japon? », dans lequel il montre qu'en Grande-Bretagne le parti impérial, celui de la plus Grande Angleterre, se heurte aux libéraux, partisans du laisser-faire et du libre-échange, hostiles au protectionnisme colonial. Miura déplore qu'il n'existe pas au Japon de parti politique prônant un «Petit Japon ${ }^{62}$ et qu'il n'existe que des partis défendant le Grand Japon. Ces derniers laissent croire que, sans expansion outre-mer, il sera impossible d'accroître la richesse et de développer le pays $^{63}$. Mais alors, fait remarquer Miura, pourquoi notre peuple ne connait-il aucune amélioration de son bien-être, voire pourquoi son niveau de vie diminue-t-il ? La vérité, c'est que l'extension outre-mer des territoires nécessite des sommes considérables pour les défendre, les maintenir et les développer, alors qu'un " Petit Japon » permettrait aux gouvernants de se concentrer sur l'amélioration des conditions d'existence et le développement des libertés. Il oppose la doctrine du Grand Japon qui propose avec l'expansion extérieure le développement du militarisme et du despotisme de l'État, à la doctrine du Petit Japon qui propose avec l'expansion conçue comme interne l'industrialisation, le libéralisme, l'individualisme $e^{64}$. Mais à aucun moment, il n'évoque le sort des colonisés. Miura se lance dans une démonstration, statistiques à l'appui, du coût que font peser les possessions coloniales sur le pays et dénonce, in fine, les illusions trompeuses de la doctrine du Grand Japon, expression que reprendra plus tard à son compte Ishibashi.

41 En 1914, ce dernier est l'un des rares à prendre position clairement contre l'entrée du Japon dans la guerre aux côtés des forces de l'Entente et proclame son hostilité à l'usage de la guerre pour obtenir des avantages territoriaux ou des gains financiers en Chine. Le dépeçage de la Chine ne peut que conduire à plus de conflictualité entre grandes puissances, écrit-i ${ }^{65}$. L'année suivante, ses prévisions se réalisent quand le gouvernement exige du gouvernement chinois ses Vingt et une Demandes. Il dénonce l'agressivité des "cliques militaires ». Les avantages économiques et militaires que le gouvernement chinois sera peut-être contraint de céder immédiatement, explique-t-il, seront annihilés sur le moyen terme par la dégradation des relations entre les deux pays et la montée inévitable en Chine d'un sentiment antijaponais qui, à terme coûtera bien plus cher au Japon que les avantages acquis. À la différence de Kōtoku Shūsui dans les années 1900 ou de Yoshino Sakuzō son contemporain, Ishibashi n'argumente pas ici au nom d'une morale ou pour dénoncer une politique dans ce qu'elle a d'excessif. Son analyse est fondée sur un pur calcul économique, sur une démarche qui se conçoit d'une objectivité quasi obsessionnelle. Il renverse la logique dominante. Il ne s'agit pas d'abandonner la Mandchourie ou Qingdao pour préserver de bonnes relations internationales ou pour faire plaisir au peuple chinois, mais pour le bien de l'État japonais. Loin des discours mystiques impériaux sur le «corps national», le kokutai 国 体, il argue qu'il faut diriger l'État comme une entreprise, c'est-à-dire, selon Ishibashi, avec un minimum de rationalité du point de vue des intérêts du Japon, sans s'embarrasser d'idéologie aberrante. Pour Ishibashi, l'impérialisme, c'est la facilité, la médiocrité, le court terme, l'absence d'ambition réelle. Il manque au Japon une réelle vision d'avenir.

42 Cette politique japonaise en Chine repose, ajoute-t-il, sur un incroyable complexe de supériorité japonais vis-à-vis des Chinois ${ }^{66}$.

Dans le vocabulaire, il n'est question que d'amitié sino-japonaise, Chine et Japon sont un même peuple, ils entretiennent des relations fraternelles comme un aîné avec un cadet [...], mais les Japonais n'ont en fait qu'une idée en tête, avaler la Chine. On ne construit pas des relations amicales sur une base pareille [...], si on 
veut vraiment construire des relations d'amitié sino-japonaises, il n'y a qu'une seule solution : laisser tomber la politique impérialiste ${ }^{67}$.

$43 \mathrm{Au}$ lendemain des mouvements antijaponais de 1919 en Corée, il explique, en contradiction complète avec l'opinion telle qu'elle s'exprime dans les principaux médias de l'époque, l'inéluctabilité de l'indépendance de la Corée :

Les Coréens forment un peuple [ichi-minzoku一民族]. Ils ont leur propre langue. Ils ont une longue histoire indépendante. Certains le regrettent sans doute, mais il n'y a pas un seul Coréen qui se réjouisse du fait que son pays soit annexé par le Japon. Jusqu'à ce qu'ils recouvrent finalement leur indépendance, les Coréens s'opposeront évidemment et de manière récurrente à la politique japonaise. Plus encore, avec le développement des connaissances et l'éveil de leur conscience, leur opposition au Japon deviendra de plus en plus radicale ${ }^{68}$.

44 Au début des années 1920, Ishibashi Tanzan s'en prend de nouveau à l'expansionnisme sur le continent chinois qui ne cherche qu'à s'emparer de privilèges économiques et manifeste une agressivité prédatrice. Il évoque l'idée d'un renoncement à une politique qui ne conduit qu'à des tensions supplémentaires avec les voisins et les grandes puissances (surtout les États-Unis), et reprend son idée de «Petit Japon». En juillet 1922, lors de la conférence de Washington, il publie éditorial sur éditorial dans son journal pour dénoncer l'impérialisme.

Et si nous abandonnions la Mandchourie, le Shandong, et si nous cessions toutes les pressions que nous exerçons sur la Chine, et si nous redonnions la liberté à la Corée et à l'île de Taiwan, quelles seraient donc les conséquences? L'Angleterre et l'Amérique seraient dans une situation impossible. Dans ce cas, ces pays ne pourraient maintenir dans le monde une posture morale et laisser seul le Japon adopter une telle politique libérale. Alors la Chine puis les petits pays dans le monde se tourneraient tous vers le Japon pour lui faire confiance. L'Inde, l'Égypte, la Perse, Haïti et les autres pays soumis aux grandes puissances, réclameraient avec force pour eux la même liberté que le Japon a donnée à la Corée et à Taiwan. Notre pays alors serait porté aux nues dans le monde entier et ni l'Angleterre, ni les ÉtatsUnis ni les autres ne pourraient rien y faire ${ }^{69}$.

Quelques jours plus tard il signe un article intitulé Dai Nihonshugi no gensō 大日本主義 の幻想 (Les illusions de la doctrine du Grand Japon), dans lequel il dénonce la médiocrité des politiques et où il s'en prend à toutes les théories fumeuses qui vantent la nécessité d'agrandir sans cesse les territoires et de relancer en permanence la course aux armements. Il raille cette mode stupide du "Grand Japon » compris comme un empire en expansion permanente ${ }^{70}$ et vante tous les avantages économiques et même politiques qu'il y aurait à renoncer à ces coûteux rêves de grandeur. "Soyons résolus à lâcher la Corée, Taiwan et Sakhaline et laissons évidemment tomber la Chine et la Sibérie " assène-t-il en introduction de ce texte provocateur dans lequel il prend position en faveur de la logique économique et de l'autodétermination des peuples ${ }^{71}$.

Après avoir souligné l'inanité des profits qu'implique l'expansionnisme outre-mer, Ishibashi pense que la volonté de résistance des peuples colonisés ou soumis brisera à terme l'empire colonial de même que l'Irlande après une lutte acharnée s'est émancipée finalement de l'Angleterre. Ishibashi ajoute qu'il est peu probable que l'Inde ne suive le même chemin.

Alors pourquoi dans ce cas, notre pays devrait-il être le seul à maintenir pour toujours sa domination sur la Corée et sur Taiwan et empêcher les Chinois ou les Russes d'exercer leur souveraineté ? Le mouvement indépendantiste coréen, le mouvement en faveur de l'établissement d'un parlement à Taiwan, les mouvements antijaponais en Chine et en Sibérie n'évoquent-ils pas déjà ce processus ? J'affirme 
que ces mouvements ne seront jamais contenus par la simple répression policière et l'intervention de troupes. C'est comme si on pensait pouvoir empêcher avec la police et l'armée le mouvement syndical des ouvriers contre les capitalistes ${ }^{72}$. et que rien ne les empêcherait. "Même si la doctrine du Grand Japon nous permettait des profits, elle ne pourrait être maintenue bien longtemps ", ajoute-t-il. Mais, en plus, elle n'est même pas une source de revenus :

$\mathrm{Au}$ lieu de construire des casernes, bâtissons des écoles, au lieu de construire des navires de guerre, bâtissons des usines. L'armée et la marine ont un budget de huit cents milliards de yens. Si déjà on pouvait investir ne serait-ce que la moitié de ce budget chaque année dans des œuvres de paix, en quelques années à peine, l'industrie japonaise changerait complètement de physionomie ${ }^{73}$.

Et plus loin :

L'abandon de la doctrine du Grand Japon, loin de nous affaiblir, nous permettra d'obtenir des gains substantiels. Si nous abandonnons des territoires comme la Corée, Taiwan, Sakhaline et la Mandchourie, et si nous faisons de la vaste Chine une amie, alors de leur propre volonté, l'Extrême-Orient dans son ensemble et tous les petits pays faibles du monde deviendront nos soutiens moraux. [...] La quintessence de la stratégie est l'harmonie. Qu'importent les armements développés par une ou deux nations arrogantes. Notre pays - leader d'un monde libéré et soutenu par les cœurs des Orientaux et du monde entier - ne pourrait plus jamais être vaincu par la guerre $^{74}$.

Ishibashi exhorte le Japon à s'efforcer « de soutenir moralement les petits pays faibles dans le monde ", c'est-à-dire tous ceux qui ne sont des "grandes puissances ». On sent chez lui quelques impatiences asiatistes et pacifistes. Une alliance entre « les peuples de couleur ", fondée sur de réelles relations de confiance et d'amitié, est la seule voie pour résister aux pressions grandissantes des pays anglo-saxons, au lendemain du traité de Washington de 1922. Pour cela, une réelle rupture avec la diplomatie traditionnelle est nécessaire.

En fait, les milieux dirigeants japonais ressentaient au cours des années 1920 de l'inquiétude devant la montée du nationalisme chinois qui aspirait à l'unification du pays, divisé depuis la proclamation de la République, en 1912, et tombé en partie aux mains des seigneurs de la guerre. Les victoires du Guomindang nationaliste leur font craindre l'émergence d'un État puissant. Pour les milieux impérialistes japonais, les progrès de la Chine vers l'unification sont un cauchemar, et Ishibashi Tanzan dénonce cette attitude visant à déclencher une guerre pour s'assurer que la partie nord-est de la Chine se détachera de manière définitive du reste du pays. Ishibashi s'oppose frontalement aux opérations militaires qui débutent en Mandchourie en septembre 1931. Quelques jours après l'« incident » du 18 septembre 1931, il publie un article au vitriol :

On ne cesse d'évoquer la «question mandchoue». Il y a bien un moyen de régler d'un coup cette fameuse question, c'est de revenir à des relations normales d'amitié entre la Chine et le Japon. Ce serait une excellente idée pour les deux pays comme pour la paix mondiale ${ }^{75}$.

50 Au lieu de calmer les dirigeants du Guomindang, les opérations de l'armée japonaise ne vont qu'exciter la fierté nationale des Chinois et un ressentiment antijaponais, pense Ishibashi. Le Japon sera entraîné, qu'il le veuille ou non, dans un conflit encore plus grave.

De même que la nation japonaise ne pourrait se résoudre à subir la domination étrangère, la nation chinoise ne peut se résoudre à pareille chose. Ceux qui se 
montrent favorables à la progression du Japon en Mandchourie refusent aux Chinois le droit de penser comme eux. Ceci ne mérite-t-il pas qu'on fasse son autocritique ? [...] La première des conditions pour que notre nation soit en mesure de régler la question mandchoue, c'est qu'elle admette tout simplement la revendication chinoise de construction d'un État unifié en Chine ${ }^{76}$.

51 La reconnaissance de la souveraineté chinoise remettrait en question l'idée que seule la poursuite des intérêts nationaux du Japon est justifiée, idée qui est au cœur même des discours nationalistes au Japon. Ishibashi ajoute que le Japon risque d'être bien isolé dans cette affaire, car heurter les sentiments nationaux des Chinois permettra à ceux-ci de s'attirer la sympathie de l'opinion internationale. Et Ishibashi de dénoncer en mai 1932 « la désinformation et la myopie étroite qui sont à l'origine de dommages incalculables pour la société japonaise » dans un pays où "il n'est plus possible de parler librement sur les relations internationales, les militaires ou quoi que ce soit qui ait de l'importance ${ }^{77}$.

Cette manière de voir le problème mandchou en tenant compte du point de vue de la Chine fit réfléchir nombre de jeunes intellectuels, notamment les jeunes sinologues de l'époque comme Takeuchi Yoshimi 竹内好 qui évoque notre "découverte ishibashienne » à la lecture ultérieure des œuvres d'Ishibashi. Takeuchi Yoshimi d'ailleurs se demande si, en ce temps-là, il y avait un seul Japonais capable de comprendre quelque chose au nationalisme chinois ${ }^{78}$.

53 Face aux exactions militaires dans les années 1930, les parlementaires japonais adoptent une attitude pour le moins ambiguë et couvrent les «crimes patriotiques » en Mandchourie. Ishibashi leur écrit sans ambages qu'«ils se rendent complices d'assassinats » et que "c'est comme si le gouvernement était composé de gangsters (bōryokudan 暴力団) ${ }^{79}$. Pour Ishibashi, l'affaire de Mandchourie a été déclenchée par des idéologues agités de motivations irrationnelles.

Ishibashi a toujours évoqué le long terme contre la pensée à court terme. Les difficultés japonaises qu'il prévoit sur le continent lui donnent aujourd'hui raison. Le Japon ne tarde pas à s'embourber en Chine, faute d'avoir analysé correctement l'ampleur de la réaction nationale chinoise. Évidemment, les mises en garde d'Ishibashi, depuis le milieu des années 1910 jusqu'au début des années 1930, sont restées lettre morte auprès des dirigeants japonais qui, pourtant, même en pleine guerre, et malgré ses opinions antigouvernementales, le consultent régulièrement. En 1937, il entre dans la Shōwa kenkyūkai 昭和研究会 qui est une sorte de think tank du Premier ministre Konoe Fumimaro 近衛文麿 (1891-1945) ${ }^{80}$ où il croise des personnalités connues de l'opposition libérale comme le philosophe Miki Kiyoshi 三木清 (1897-1945). Mais c'est bien Ishibashi qui a raison sur le fond: le « Grand Japon » n'a aucun avenir historique. Il parle contre un gouvernement des militaires, pour le retour à un régime parlementaire. Après Pearl Harbor, il écrit: "je suis un libéral, mais je ne suis pas un traître à l'État » et critique l'extension permanente du théâtre des opérations militaires ${ }^{81}$. On le sent au désespoir de voir son pays s'engager dans une entreprise dont il pronostique l'échec final.

La croissance phénoménale du Japon dans les années 1950-1960 vient donner raison à Ishibashi a posteriori, lorsqu'il prônait un «Petit Japon » recentrant son développement sur une croissance interne, loin des rêves impérialistes. Yoshida Shigeru 吉田茂 (1878-1967), Premier ministre de l'après-guerre, confie à Ishibashi Tanzan le soin de la reconstruction économique du pays en le nommant ministre des Finances (mai 1946mai 1947). Hostile à la réforme économique prônée par les autorités d'occupation, 
notamment la dissolution des zaibatsu, il entre en conflit avec les autorités américaines, car il veut aussi réduire les frais qu'implique le stationnement des forces alliées. Malgré sa sourde opposition au régime militariste pendant la guerre, Ishibashi est alors victime de l'épuration imposée par les Américains qui ne lui pardonnent pas de leur avoir résisté, et il doit renoncer à toute fonction publique pour un temps. Mais il n'en devient pas moins l'un des leaders du parti libéral-démocrate, ministre du Commerce international et de l'Industrie en 1954-56, pousse au rétablissement de relations avec l'URSS et la Chine communiste ${ }^{82}$, puis finalement est nommé Premier ministre (de décembre 1956 à février 1957), malgré la franche opposition des Américains ${ }^{83}$. Malade, il doit abandonner son poste à peine deux mois après sa nomination.

Ishibashi Tanzan est un esprit précurseur et visionnaire. Sur le plan économique, c'est un libéral (mais c'est aussi l'introducteur de Keynes au Japon), sur le plan politique un démocrate plutôt modéré opposé au communisme (mais résolument opposé à l'expansionnisme militaire et au fascisme), sur le plan diplomatique un partisan d'un " petit Japon » (mais il hésite entre isolationnisme et un rôle moral de « leader global » pour son pays). Son anticolonialisme n'est pas un anticolonialisme de solidarité avec les colonisés, mais plutôt une position de principe. Quand on le relit aujourd'hui, on est frappé par son raisonnement toujours lucide. Jamais il ne cède aux sirènes de son temps. Persuadé qu'on ne gagne jamais d'argent très longtemps en faisant la guerre, sa position le conduit à entrer en conflit avec les milieux impérialistes et colonialistes d'avant 1945 qu'il conçoit aussi comme des éléments antidémocratiques. Ses positions sont tellement lucides que certains essayistes se sont même amusés à imaginer ce que dirait Ishibashi du Japon d'aujourd'hui et comment il critiquerait les dirigeants japonais, exercice, certes, totalement vain, mais qui repose sur une grande admiration pour un personnage atypique ${ }^{84}$. On est frappé de constater à quel point ses positions dans les années 1920 et 1930 préfigurent ce que sera le Japon des années de la Haute Croissance, "un géant économique et un nain politique », un pays pacifique dont le niveau de vie moyen augmente avec une grande régularité sans intervenir dans les affaires des autres peuples d'Asie.

\section{Doutes académiques sur le bien-fondé du colonialisme}

57 Mais Ishibashi n'est pas le seul à développer des analyses économiques critiques sur l'impérialisme et le colonialisme. À dire vrai, la question de la politique coloniale est devenue l'objet d'un discours à prétentions scientifiques. À partir de 1895, elle devient l'enjeu d'une réflexion, car les premiers administrateurs « éclairés » de Taiwan pensent trouver dans une "démarche scientifique" les solutions aux problèmes auxquels ils sont confrontés et Gotō Shinpei veut vraiment faire de la nouvelle colonie un " laboratoire ${ }^{85}$. À partir de la victoire de 1905 sur les Russes, le Japon change de statut international et émerge comme une nouvelle "grande puissance ». À cette occasion, rappelons qu'il obtient la partie méridionale de Sakhaline, des zones à bail dans la péninsule du Liaodong, exerce son protectorat en Corée ainsi qu'une forte influence en Mandchourie. Aussi, dès le lendemain de la guerre russo-japonaise, l'étude académique des politiques coloniales s'impose dans les universités comme objet de recherches, comme savoir théorique et pratique. Dès 1903, Nitobe Inazō, le fameux auteur de Bushido, Soul of Japan, avait été nommé chargé de cours, à l'Université Impériale de 
Kyōto, à son retour d'un séjour à Taiwan où il avait fait l'expérience de l'administration coloniale $^{86}$. La première université à instituer une chaire d'études coloniales, c'est l'Université Impériale du Tōhoku, à Sendai, dès $1907^{87}$. Puis, en 1909, Nitobe prend la nouvelle chaire à l'Université Impériale de Tōkyō. Yanaihara Tadao lui succède en $1922^{88}$. Il n'a pas encore trente ans.

Yanaihara fait partie de ce courant chrétien japonais pacifiste, non affilié à une église, proche d'Uchimura Kanzō 内村鑑三 (1861-1930), le grand intellectuel chrétien de la fin du XIX et du début du Xxe siècle, lui-même influencé par le pacifisme sans compromis de Léon Tolstoï. Yanaihara Tadao se dit impressionné par le côté entier et indépendant d'Uchimura Kanzō. Il est par ailleurs proche de personnalités chrétiennes qui ont rejoint le courant du socialisme modéré dont Kinoshita Naoe 木下尚江 (1869-1937) ou Abe Isoo sont les représentants les plus éminents. À l'université, les personnalités de Nitobe Inazō et de Yoshino Sakuzō le fascinent.

Au pacifisme moral dont il s'inspire, Yanaihara Tadao ajoute un anticolonialisme assez radical en son temps, fondé sur une analyse économique et politique documentée qui, comme dans le cas d'Ishibashi Tanzan, se veut rationnelle. Il collabore à la revue Chūō kōron. Il publie des ouvrages critiques sur Taiwan, la Corée, la Mandchourie. En 1935, il donne une étude sur les îles du sud sous domination japonaise et, en 1937, un travail sur l'Inde sous la domination britannique. Dès 1937, son opposition radicale à la nouvelle guerre menée en Chine vaut à Yanaihara une interdiction professionnelle qui le fait expulser de l'Université Impériale de Tōkyō. Après la guerre en novembre 1945, il est réintégré comme professeur à l'Université de Tōkyō, dont il devient le recteur, de 1951 à 1957. L'historien de l'économie occidentale, Ōtsuka Hisao 大塚久男 (1907-1996), le chef de file dans l'après-guerre - avec Maruyama Masao 丸山眞男 (1914-1996) -, du courant «moderniste ", se disait lui-même disciple de Yanaihara, lequel émerge alors comme une sorte de figure intellectuelle et morale ${ }^{89}$.

Vers 1920, Yanaihara prend des positions franchement hostiles à la répression à laquelle se livrent le gouvernement et l'armée en Corée. Humaniste et démocrate, il dénonce la politique japonaise qui appauvrit les paysans coréens et les jette dans la misère et plaide pour l'autonomie coloniale des colonies dans le cadre de l'empire. En 1926, dans un texte qu'il publie dans Chūō kōron, il montre que la répression a, certes, vaincu les manifestants en 1919, mais que ceux-ci, c'est-à-dire le peuple coréen dans son ensemble, ont remporté une victoire ${ }^{90}$. Il ajoute que, malgré la volonté de changement, le peuple coréen est victime d'une politique fiscale qui l'étrangle, tandis que les terres sont confisquées peu à peu par les colons japonais. Il s'agit là d'un processus qui confisque aux producteurs leurs moyens de production et prolétarise le pays, laissant aux Coréens tout juste de quoi survivre, et contraignant beaucoup d'entre eux à émigrer vers la Sibérie ou la Mandchourie et même, plus récemment, vers la métropole ${ }^{91}$. La politique japonaise d'exportation du riz de la péninsule vers l'archipel oblige les paysans coréens à s'échiner à produire pour la métropole et à se contenter de maigres repas ${ }^{92}$. Dans les tréfonds de la société coréenne, on perçoit une «instabilité désespérée " écrit-il à plusieurs reprises ${ }^{93}$. Yanaihara juge à ce stade normal qu'on octroie le droit pour les Coréens de participer à la vie politique et administrative de leur pays, en clair le droit de vote (les Japonais n'ont obtenu eux-mêmes le suffrage universel - pour les hommes - que l'année précédente), soit en les laissant participer à la vie politique de la métropole, mais surtout en créant un parlement qu'il faudrait instituer à Séoul ${ }^{94}$. «De même que la classe ouvrière peut suffisamment défendre ses 
propres intérêts en envoyant ses représentants au parlement, les colonisés pourront défendre leurs propres intérêts en participant eux-mêmes aux décisions politiques " $^{95}$.

61 Yanaihara décrit la politique coloniale comme suit : il s'agit d'un régime d'exploitation de nature «despotique»(sensei seiji 専制政治) qui ne reconnait aucun droit aux colonisés, traités comme s'ils étaient des « Noirs sous développés» (mikai kokujin 未開 黒人) ${ }^{96}$, qui pratique une politique d'assimilation et qui se fonde sur une administration coloniale autonome. Or, dit-il, le régime d'exploitation est le produit d'une époque: violence impérialiste et revendications démocratiques qui traversent les sociétés japonaise et coréenne se reflètent dans les orientations en matière de politique coloniale. Il incite le parlement japonais à exempter les colonisés du paiement de nouveaux impôts, et il prône la libération de « ceux qu'on torture et qu'on étouffe ». Il faut avoir, écrit-il, le courage de voir les choses en face ${ }^{97}$. Quant à l'objectif de l'assimilation - si tant est qu'il soit possible -, il est irréalisable s'il doit être le fait d'une administration coloniale qui constitue en soi un obstacle à l'assimilation. Si le Japon devait s'appuyer sur pareille administration, la collaboration avec les Coréens deviendrait vite impossible, car leurs pratiques sociales sont elles-mêmes le produit d'une histoire différente de celle du Japon ${ }^{98}$.

62 À ce stade, l'indépendance de la Corée ne fait pas encore partie des options envisagées pour Yanaihara. Il parle en faveur de l'autonomie politique des habitants de la péninsule, des garanties démocratiques pour les Coréens, mais ne va guère au-delà. En ce sens, il reste encore proche de son collègue de Kyōto, Yamamoto Miono, qui avait été un actif partisan pendant la Première Guerre mondiale de la transformation des possessions allemandes du Pacifique en colonies japonaises, mais qui développait de son côté le refus de la politique d'assimilation et se montrait partisan de l'autogouvernement des colonisés (jichi shugi 自治主義) ${ }^{99}$. Yanaihara pense qu'il faut venir à bout de la résistance des Coréens, mais sans s'aliéner les populations, plutôt en les convainquant de l'alliance nécessaire entre les deux peuples dans le cadre de l'empire. D'ailleurs le home rule en faveur duquel il se prononce est pour lui le meilleur rempart pour éviter que les Coréens ne s'émancipent et ne se séparent complètement du Japon ${ }^{100}$. Il va plus loin encore en expliquant que si les Coréens devaient s'émanciper et devenir indépendants dans ce cadre, ce serait un grand succès pour la politique coloniale du Japon et «l'honneur de l'empire » ${ }^{101}$.

Dans un ouvrage critique sur le colonialisme japonais à Taiwan, Taiwan sous l'impérialisme publié en 1929, réédité en collection de poche en 1937, Yanaihara livre sans doute la première étude solide en langue japonaise sur Taiwan, rédigée d'un point de vie critique ${ }^{102}$. L'impact de l'ouvrage est d'autant plus fort que l'auteur est professeur d'études coloniales à l'Université Impériale de Tōkyō. D'ailleurs cette étude sur Taiwan sera souvent considérée après 1945 comme le point de départ des area studies japonaises (chiiki kenkyu 地域研究) et Kuwabara Takeo la considère comme un classique des sciences sociales japonaises, évoquant un "ouvrage d'une grande rigueur scientifique dont nous autres Japonais pouvons être fiers $»^{103}$. Au moment de sa parution, l'ouvrage est interdit d'exportation à Taiwan, mais il devient la bible des étudiants taiwanais qui viennent faire leurs études au Japon où il reste disponible dans les bibliothèques et connaît une première traduction en chinois sur le continent dès $1930^{104}$. Yanaihara explique que les grandes sociétés capitalistes japonaises comme Mitsui ou Mitsubishi ont monopolisé les industries de l'île (l'agroalimentaire et l'industrie sucrière surtout), et que les paysans et les ouvriers taiwanais sont les victimes d'une oppression et d'une 
exploitation sociale et économique féroce. Son argumentation s'appuie sur une forme d'économisme (proche du marxisme en l'occurrence). Surtout, et c'est là peut-être le point central, Yanaihara indique clairement la nécessité de prendre en compte les revendications du mouvement national taiwanais.

Yanaihara commence par retracer l'histoire de l'île, Formosa, la Magnifique, que se disputèrent au XVII siècle Portugais, Hollandais et Chinois. Les Chinois l'emportèrent, mais au XIX ${ }^{e}$ siècle, l'île est de nouveau l'objet de la convoitise entre Prussiens, Américains et Français avant que les Japonais ne s'en emparent définitivement, cédée par Pékin à Tōkyō à l'issue de la guerre sino-japonaise ${ }^{105}$. Yanaihara revient à cette occasion sur l'analyse de la guerre sino-japonaise de 1894-1895, qu'il présente non comme "une guerre nationale » comme le veut la propagande officielle, mais déjà comme « une guerre impérialiste $»^{106}$.

$\mathrm{Au}$ Japon vers 1895 où le capitalisme industriel était encore immature, l'impérialisme était déjà solidement ancré comme idéologie ${ }^{107}$. En fait, l'occupation de l'île a coûté bien plus cher que les profits qui en ont été tirés, et Yanaihara estime qu'il s'agit là de "dépenses de luxe » que s'est offert l'État japonais au nom d'une vision stratégique discutable ${ }^{108}$. En s'emparant de Taiwan pour éviter que les autres grandes puissances ne le fassent, le Japon a adopté là, pour la première fois clairement, une attitude impérialiste. Le choix n'avait pourtant rien d'inéluctable. Mais la critique de Yanaihara porte, comme celle d'Ishibashi, sur les résultats : dépenses (publiques) plus élevées que les profits (privés), et dégradation de l'image symbolique du pays dans la région. Au bout du compte, Yanaihara se demande quel prix le Japon aurait pu tirer de Taiwan s'il l'avait revendu et s'il avait investi les sommes gagnées dans les circuits de l'économie nationale ${ }^{109}$. Sans employer l'expression de «Petit Japon », Yanaihara est ici assez proche des positions défendues par Ishibashi Tanzan.

Yanaihara critique aussi « le caractère exacerbé du despotisme colonial, rarement égalé dans les autres expériences mondiales de colonisation ${ }^{110}$, écrit-il, non sans exagération, sans doute trop rapidement ${ }^{111}$. La brutalité de l'entreprise coloniale combinée par ailleurs à des investissements publics conséquents a bien fini pourtant par produire des résultats certains, notamment dans le domaine de l'hygiène publique, des infrastructures et des transports. Ces succès d'ailleurs rendent inéluctable la nécessité de réformes politiques, sans quoi le mouvement de résistance à la japonisation de l'île ne cessera de croître. Il conclut au caractère contradictoire du développement de l'impérialisme colonial, qui s'empresse vers sa propre perte, en quelque sorte ${ }^{112}$.

D'une façon générale, Yanaihara est hostile aux politiques assimilationnistes car il pense que le sort des Japonais n'est pas si enviable, compte tenu des pratiques autoritaires et de la faiblesse de la démocratie en métropole. Mais dans les deux cas que représentent Taiwan et la Corée, les problèmes sont identiques au-delà des différences historiques. Il faut donner des droits politiques aux populations colonisées de manière à les laisser s'administrer elles-mêmes. Et si elles réclament l'indépendance, il faut la leur accorder, pense Yanaihara, car elles ne pourront alors qu'entretenir des relations d'amitié et de paix avec l'ancienne métropole. Il n'y a donc rien à perdre, à terme, d'une indépendance des colonies si le processus est maîtrisé pacifiquement. Les positions de Yanaihara sont donc fort critiques des politiques japonaises traditionnelles. Il affirme un réformisme assez radical, mais ne franchit jamais le pas de la rupture entre la métropole et les colonies. 
68 À ce titre d'ailleurs, il est l'objet de critiques de la part de ceux qui sont proches du marxisme. Dans Impérialisme, stade suprême du capitalisme (1916), Lénine est l'un des premiers à avoir explicitement fait le rapport entre impérialisme et colonialisme, mais son analyse ne fut guère connue au Japon avant les premières années de la décennie 1920. On sait que Yanaihara commente l'ouvrage de Lénine dans ses cours ${ }^{113}$. L'un de ses anciens collègues de la Faculté d'Économie, Hosokawa Karoku 細川嘉六 (1888-1962), qui travaille à l'Institut des Questions Sociales Ōhara (Ōhara shakai mondai kenkyūjo 大原社会問題研究所) s'interroge ouvertement sur la contradiction inhérente à la fonction qu'occupe Yanaihara lui-même, à laquelle « on ne peut qu'être l'avocat des intérêts de classe des capitalistes ", le porte-parole d'un système et il émet des doutes sur le caractère scientifique de pareils enseignements ${ }^{114}$. Plus tard, les tenants de l'école marxiste kōza développent, en 1932-1933, une critique radicale du colonialisme associé intrinsèquement au capitalisme, et à l'impérialisme dans les «thèses sur l'histoire du développement du capitalisme japonais » (Nihon shihonshugi hattatsushi kōza 日本資本主 義 発達史講座). Mais, parce qu'ils considèrent le colonialisme comme un épiphénomène de l'impérialisme, ils omettent de proposer une étude critique particulière du système dont ils pensent qu'il s'effondrera de lui-même, avec la révolution à venir au Japon ${ }^{115}$. Le seul à avoir tenté une analyse marxiste de la situation, à Taiwan, incluant une critique radicale du colonialisme, c'est Yamakawa Hitoshi ${ }^{116}$. Selon lui, après des débuts difficiles, la colonie est devenue rentable à partir de 1908. Il s'agit donc d'un succès, mais pour qui ? Pas pour les colonisés qui sont victimes d'une exploitation économique doublée d'une dure domination politique. Il montre la confiscation des terres par les industries du sucre et du papier, entièrement contrôlées par le capital métropolitain, avec l'appui politique de l'administration, la discrimination salariale entre colons et colonisés. Il évoque aussi comment la mise au point d'un droit spécial, l'article de loi 63, permet au gouverneur général de se comporter en "souverain despotique » et comment le passage à une administration civile, depuis 1920, n'a pas modifié réellement les choses, les lois de répression policière restant en vigueur. Il prend position en faveur de la montée d'un mouvement démocratique à Taiwan et de l'acquisition des mêmes droits pour les colonisés et les métropolitains, mais s'inquiète en définitive de l'indifférence fondamentale des prolétaires japonais pour la situation dans les colonies ${ }^{117}$.

Yanaihara reprend, dans sa propre étude, bien des aspects de l'analyse économique marxiste développée par Yamakawa Hitoshi, mais il ne se rallie jamais aux valeurs du communisme. Yanaihara propose, non pas un simple panorama critique des politiques coloniales, mais de lancer des recherches sous la forme d'enquêtes de terrain concrètes sur les sociétés coloniales. Son travail possède d'ailleurs une dimension pluridisciplinaire certaine, dans lequel sont associées l'histoire, la politique, la sociologie, l'éducation et l'étude économique. C'est d'ailleurs bien dans ce sens qu'on peut le considérer comme un pionnier des études portant sur les aires culturelles.

Quelques mois après le coup de force de septembre 1931 en Mandchourie, Yanaihara écrit un article, qu'il reprendra plus tard dans $L a$ Question mandchoue ${ }^{118}$. Pour comprendre la situation de la Mandchourie, Yanaihara retrace les rivalités impérialistes dans la région depuis le début $\mathrm{du} \mathrm{xx}^{\mathrm{e}}$ siècle, et en vient à décrire les causes des mouvements antijaponais en Chine qu'il présente d'une part comme « une nécessité historique ", eu égard au développement économique et politique de la Chine et de la Mandchourie, et d'autre part comme un «mouvement national». Pour lui, ce 
mouvement national antijaponais est profond et probablement impossible à endiguer. L'idée de protéger les intérêts particuliers et les privilèges du Japon en Chine ne peut qu'aboutir à un choc avec le nationalisme chinois qu'il considère comme un mouvement ascendant. Dans ces conditions, la politique du Japon en Mandchourie ne peut qu'aboutir à une aggravation de la situation. À partir de là, il réfute les discours colonialistes qui justifient la présence japonaise dans la région et qui s'appuient sur trois arguments économiques, l'émigration, les profits tirés du commerce et l'idée du bloc commercial nippo-mandchou.

71 L'émigration de paysans japonais en direction de la Mandchourie ne résoudra pas la question du surplus démographique en métropole, explique Yanaihara. Chassés par la misère au Japon, les colons qu'il assimile à des émigrants se retrouvent sur place dans une situation difficile avec, pour eux, des frais d'installation considérables et, pour le pays, des frais généraux pour assurer le maintien de l'ordre et l'approvisionnement d'un marché plus dispendieux que les profits à escompter. L'invasion japonaise a coupé les liens commerciaux traditionnels entre la Mandchourie et la Chine et crée des perturbations dans les circuits économiques. Ceux-ci se reconstituent avec le Japon, mais la faiblesse structurelle du marché intérieur mandchou pèse très peu dans le commerce extérieur japonais, guère plus de 5 à $10 \%$, ce qui n'est rien par rapport aux dépenses engagées pour le contrôle du territoire et le maintien de l'ordre. D'autant que la situation se dégrade rapidement avec la Chine, comme conséquence de la prise de pouvoir sur la Mandchourie. Dès 1932, Yanaihara pronostique une nouvelle guerre sino-japonaise, qui éclatera en effet, en 1937. Enfin, la constitution d'un bloc économique nippo-mandchou autosuffisant, prôné par certains cercles, est-elle réalisable et serait-elle réellement efficace ? L'idée serait de puiser dans le territoire mandchou qui recèle des matières premières en grande quantité. Mais, explique Yanaihara, ces ressources, si importantes qu'elles soient, sont insuffisantes pour une économie comme le Japon. En outre, elles sont souvent de qualité médiocre ou d'extraction peu rentable. En conclusion, Yanaihara pense que l'occupation de la Mandchourie, considérée du seul point de vue de la rentabilité globale, constitue une erreur économique grave et coûteuse. Elle ne rapporte, explique-t-il, qu'à court terme et qu'aux capitalistes qui ont investi dans la région. Plus gravement encore, elle implique une guerre difficile contre la Chine, qui coûtera à la Nation bien plus que l'occupation de la Mandchourie ne rapportera jamais, et dont l'issue est aléatoire.

72 Sous couvert d'analyse politique, Yanaihara se livre à une critique de l'argumentation impérialiste développée abondamment dans la presse de son temps. Économiquement, la colonisation ne servira à rien. Mais plus grave encore, vouloir construire politiquement la prospérité de la nation japonaise sur l'oppression de la nation chinoise, elle-même engagée dans sa propre construction, revient à ne rien comprendre au sens des mouvements politiques et sociaux qui animent l'Asie depuis le début du siècle. L'échec des prétentions japonaises est quasiment assuré, écrit-il. "À l'inverse, si le Japon reconnaissait la volonté chinoise de créer un État unifié, l'aidait à réaliser cet objectif, il s'aiderait lui-même et contribuerait à la paix en Asie $»^{119}$.

À partir de là, la question se pose aussi en termes moraux. L'invasion est-elle par ailleurs juste? Non, et ce aussi pour des raisons de principe :

Il est juste de faire obstacle à l'invasion [de la Mandchourie par le Japon]. Il est juste de ne pas provoquer la guerre pour cela. La justice exige, par d'autres moyens que la guerre, de mettre fin à l'invasion. L'objectif de la justice c'est d'empêcher la guerre, 
de châtier ceux qui ont déclenché l'invasion. Et la méthode de la justice, c'est aussi la paix ${ }^{120}$. en leur temps, des leaders d'opinion. Mais ils ne sont pas seuls. Il est d'ailleurs intéressant de noter, au passage, que les professeurs chargés, dans les universités impériales, de l'étude des sociétés coloniales sont, en grande partie, hostiles aux brutales politiques colonialistes développées par les différents gouvernements japonais. Dans un article publié dans Taiyō en mai 1920, Yamamoto Miono qui enseigne les politiques coloniales à l'Université de Kyōto parle en faveur d'une administration locale gérée par les Coréens eux-mêmes, et de députés représentant les minorités coloniales au parlement à Tōkyō. D'autres prennent la parole, au contraire, en faveur du projet assimilationniste au nom de la démocratie. C'est notamment le cas de Uehara Etsujirō 上原悦二郎 (1877-1962), qui rêve d'un Japon où la révolution démocratique aurait été accomplie, et qui serait capable d'assimiler une péninsule coréenne à son tour démocratisée, pour construire une Nation commune qui ignorerait les discriminations $^{121}$.

Le colonialisme antirépressif et réformiste des démocrates japonais des années 1920 et 1930 peut paraître aujourd'hui désuet. Qu'on ne s'y trompe pas. D'une part, bien rares étaient ceux qui, en Grande-Bretagne ou en France, par exemple, dénonçaient vers la même époque aussi clairement les abus du colonialisme. Les critiques japonais du colonialisme japonais sont, de ce point de vue, tout à fait remarquables. Par ailleurs, les radicaux indépendantistes étaient alors minoritaires dans les colonies japonaises. En effet, une partie des nationalistes coréens ou taïwanais pensaient sincèrement - à l'instar des démocrates japonais - que le système colonial était réformable. C'est ainsi qu'en 1921, le député Tagawa Daikichirō, l'un des défenseurs de la «démocratie impériale » comme la pense Yoshino Sakuzō, présente au parlement une pétition taiwanaise en faveur de la création d'un parlement à Taipei. Elle est signée de Taiwanais qui se présentent comme « des Japonais aspirant eux aussi à la démocratie ». Des pétitions de ce type seront présentées de très nombreuses fois à Tōkyō. De même, dès 1920, des pétitions de même style circulent en Corée en faveur d'une loi permettant aux Coréens d'être éligibles aux élections. Le droit de participer à la vie publique est une revendication fondamentale des élites locales taiwanaises et coréennes. Prenant les assimilationnistes japonais au pied de la lettre, ils se déclarent eux-mêmes "Japonais " ou « nationaux » et c'est à ce titre qu'ils interpellent les autorités japonaises sur place ou en métropole en exigeant une représentation à la capitale ${ }^{122}$.

D'une certaine façon, on peut dire que les nationalistes taiwanais ou coréens sont euxmêmes pris dans la vague démocratique qui a déferlé sur le Japon à partir de 1918, qu'ils en sont partie prenante, et qu'ils revendiquent plus de démocratie ou d'autonomie dans leur pays comme en métropole. Un début de convergence naît alors entre libéraux et démocrates japonais et certaines élites locales dans les sociétés colonisées pour lutter contre les systèmes répressifs et brutaux mis en place par l'État colonial.

Dans un Japon où les partisans de l'expansionnisme sont majoritaires, les voix qui s'expriment pour critiquer le colonialisme restent finalement limitées. Issues de l'élite intellectuelle, elles ne sont pourtant guère écoutées dans les sommets de l'État où les factions militaristes et impérialistes pèsent d'un poids trop lourd. Et plus ces factions pèsent, plus elles rendent la critique difficile voire impossible. Mais n'imaginons pas 
non plus que les mouvements anticolonialistes ont pesé très fort dans les métropoles coloniales occidentales avant la Seconde Guerre mondiale. C'est l'ampleur prise après la guerre par les mouvements anticolonialistes dans les colonies elles-mêmes qui, le plus souvent, déclenchent à leur tour une prise de conscience de la réalité coloniale dans les métropoles. Les mouvements anticolonialistes n'ont pu prendre leur essor au Japon parce que, dans le cas japonais, la décolonisation intervient avec la défaite militaire, d'un coup en quelque sorte, en août-septembre 1945. Après tout, la France vaincue de 1940 ne perd pas pour autant son empire colonial. Or le Japon se retrouve, après 1945, sinon dans une position de colonisé, du moins dans une position de vaincu et de pays soumis à la présence de l'armée américaine sur son propre sol. C'est un paradoxe de ce pays que d'avoir été contraint de vider le discours anticolonialiste de toute pertinence après 1945. Comme le fait remarquer Nishikawa Nagao, les Japonais d'après-guerre se perçoivent autant comme anciens colonisateurs vis-à-vis de leurs anciennes colonies qu'ils se sentent colonisés ou semi-colonisés par les États-Unis. Il évoque des mentalités qui naissent alors et font des Japonais des « colonisés de l'intérieur » ${ }^{123}$.

Des critiques percutantes du régime colonial avaient été exprimées - certes de manière minoritaire - avant la décolonisation. De ce point de vue, le mouvement de la société japonaise n'est pas en décalage avec les pays occidentaux. D'ailleurs, dans les métropoles coloniales d'Occident, peu nombreux étaient, avant les années 1930, les discours anticolonialistes un tant soit peu articulés. Claude Liauzu évoque à propos de la France d'avant-guerre la "marginalité de l'anticolonialisme $»^{124}$. Cette capacité de certains critiques japonais à se hisser au-dessus de la mêlée, à alerter sur la catastrophe à venir, montre, s'il fallait le rappeler, la capacité de discernement et la lucidité d'une partie de la "société civile" japonaise. Elle suggère en même temps certaines continuités, par-dessus la phase du militarisme et de la guerre, entre les politiques libérales et démocratiques avant et après-guerre.

\section{NOTES}

1. Selon l'expression de l'économiste et spécialiste des politiques coloniales Yanaihara Tadao 矢 内原忠雄 (1893-1961). Sur les grandes lignes de la politique coloniale japonaise, voir Alain Delissen, «La Corée », in Hartmut O. Rotermund et al., L'Asie Orientale et Méridionale aux XIX et XXe siècles, Paris, Puf, Nouvelle Clio, 1999, 546 p., p. 135-232, notamment le chapitre III «Le premier $\mathrm{xx}^{\mathrm{e}}$ siècle : les ambivalences de la colonisation japonaise », p. 177-195; voir également Pierre-François Souyri, «La Colonisation japonaise: un colonialisme moderne mais non occidental », dans Marc Ferro (dir.), Le Livre noir du colonialisme, XVII ${ }^{e}-\mathrm{XXI}{ }^{e}$ siècle : de l'extermination à la repentance, Robert Laffont, Paris, 2003, 1124 p., p. 407-430. En anglais, voir notamment Ramon H. Myers and Mark. R. Peattie, The Japanese Colonial Empire, 1895-1945, Princeton University Press, 1984, 560 p., qui fait toujours autorité.

2. Nous évitons le terme "impérialisation» qui correspond plus à l'esprit de l'expression japonaise et est utilisé par les auteurs anglophones (imperialization) mais n'est décidément guère correct en langue française. 
3. «Discours sur les ancêtres communs des Japonais et des Coréens» (Nissen dōsoron 日鮮同祖 論). Une mise au point sur ces questions dans Oguma Eiji 小熊英二, Tan.itsu minzoku shinwa no kigen 単一民族神話の起源 (Les Origines du mythe du peuple homogène), Shin.yōsha 新曜社, 1995, 450 p., notamment les chapitres 5 et 13. Voir aussi Arnaud Nanta, Débats sur les origines du peuplement de l'archipel japonais dans l'anthropologie et l'archéologie (décennie 1870 - décennie 1990), Université Paris 7 (thèse non publiée), 2005.

4. Oguma Eiji, op. cit., p. 239.

5. Itagaki Taisuke, partisan de "l'harmonie des races" sera l'instigateur en 1914 d'un projet avorté de conférer aux habitants de Taiwan les mêmes droits que les Japonais. Cf. Mark R. Peattie, "Japanese Attitudes Toward Colonialism", in Myers and Peattie (dir.), The Japanese Colonial Empire, op.cit., p. 103.

6. Sur cette affaire dite "incident d'ōsaka ", voir Lionel Babicz, Le Japon face à la Corée à l'époque Meiji, Maisonneuve \& Larose, 2002, p. 148-154.

7. Tsurumi Shunsuke 鶴見俊輔, Senjiki Nihon no seishinshi 1931-1945 戦時期日本の 精神史 (Histoire psychologique du Japon en guerre, 1931-1945), Iwanami shoten 岩波書店, 1982, réed. 2001, 296 p., p. 121. On notera cependant que peu après l'annexion des Ryūkyū, Ueki Emori 植木 枝盛 (1857-1892), futur leader du Mouvement pour les droits du peuple et la liberté, avait publié un article intitulé: «De la nécessité de donner son indépendance aux Ryūkyū » (Ryūkyū no dokuritsu seshimubeki ronzu 琉球の独立せしむべき論ず), in Aikokushirin 愛国志林, Ōsaka, mars 1880, texte repris dans Itō Teruo 伊東昭雄, Ajia to Kindai Nihon, Hanshinryaku no shisō to undō アジアと近代日本一反侵略の思想と運動 (L'Asie et le Japon moderne. La pensée et les mouvements hostiles à l'invasion), Shakai hyōronsha 社会評論社, 1990, 316 p., p. 16-19.

8. Cité par Yanaihara Tadao, Teikokushugi ka no Taiwan, seidoku 帝国主義下の台湾精読 (Taiwan sous l'impérialisme, exégèse), édition mise au point par Wakabayashi Masahiro 若林正丈, Iwanami bunko 岩波文庫, 2001, p. 35. Takekoshi Yosaburō fut vers 1890 l'un des membres les plus actifs de la Minyūsha 民友社, la Société des amis du peuple, un nouveau courant à la fois nationaliste et démocrate. En 1905, il était député du parti Seiyūkai 政友会, proche du futur Premier ministre Saionji Kinmochi 西園寺公望 (1849-1940).

9. Kōtoku Shūsui, L'Impérialisme, le spectre $d u x^{e}$ siècle, traduit, présenté et annoté par Christine Lévy, CNRS éditions, 2008, 188 p. La vulgate de la recherche consacrée à Kōtoku Shūsui a largement montré sa fascination pour les mouvements socialistes et anarchistes européens et américains. Ce point de vue est partiellement remis en question par l'historien Ishimoda Shō 石 母田正 (1912-1986), Kōtoku Shūsui to Chūgoku, minzoku to aikokushin no mondai ni tsuite 幸徳秋水と 中国一民族と愛国心の問題について (Kōtoku Shūsui et la Chine, Sur la question du peuple et du patriotisme), texte de 1952, republié dans Takeuchi Yoshimi (dir.), Ajia shugi アジア主義 (L'Asiatisme), tome 9 de Gendai Nihon shisō taikei 現代日本思想体系 (Anthologie de la Pensée japonaise de notre époque), Chikuma shobō 筑摩書房, 1963, 444 p., p. 384-410. Dans ce texte, Ishimoda cherche à montrer le sentiment de solidarité que Kōtoku aurait éprouvé pour la lutte pour l'indépendance des peuples colonisés.

10. Pour reprendre le terme employé par Andrew Gordon, dans son ouvrage Labor and Imperial Democracy in Prewar Japan, University of California Press, 1991, 384 p.

11. Matsuo Takayoshi 松尾尊 (textes établis et annotés par), Yoshino Sakuzō shū 吉野作造集 (Recueil des œuvres de Yoshino Sakuzō), tome 17 de Kindai Nihon shisō taikei 近代日本思想体系 (Anthologie de la pensée japonaise moderne), Chikuma Shobō, 1976, 497 p., postface de Matsuo Takayoshi, p. 474. Par autodétermination, il faut plutôt entendre ici une certaine latitude accordée aux colonisés pour gérer leurs propres affaires selon leurs propres coutumes.

12. Et ce, bien qu'il ait été de 1904 à 1906 le répétiteur privé du fils de Yuan Shikai 袁世凱, le futur rival conservateur de Sun Yat Sen (Song Zhongshan 孫中山) à la tête de la nouvelle Chine républicaine. 
13. Reproduite dans Yoshino Sakuzō sensh̄̄ 吉野作造選集 (Euvres choisies de Yoshino Sakuzō), tome 7, Iwanami shoten, 1995, $418 \mathrm{p}$.

14. «Taishigaikō konponsaku no kettei ni kansuru Nihon seiryaku no konmei » 対支外交根本策 の決定に関する日本政略の混迷 (Les errements de stratégie du Japon dans les décisions prises sur les orientations fondamentales de la politique vis-à-vis de la Chine), publié en 1916 dans la revue Chūō kōron 中央公論. Voir la réédition dans Yoshino Sakuzō shū, op. cit. p. 134-135.

15. La kenpeitai japonaise a souvent été comparée par la suite à la gestapo allemande pendant la Seconde Guerre mondiale.

16. Yoshino Sakuzō, «Mankan o shisatsu shite» 満韓を視察して (En observant la Mandchourie et la Corée), Chūō kōron, juin 1916, dans Yoshino Sakuzō shū, op. cit., p. 145, cité par Narita Ryūichi 成田龍一, Taishō demokurashii 大正デモクラシー (La Démocratie de Taishō), Iwanami shinsho, 2007, 258 p., introduction p. iv.

17. «Chōsen tōchisaku » 朝鮮統治策, (La domination de la Corée) octobre 1918, publié dans Chūō kōron, dans Yoshino Sakuzō, senshū, op. cit., tome 9, p. 50-51. Sur Yamamoto Miono, voir infra.

18. Il s'agit du mouvement qui éclate $1^{\mathrm{er}}$ mars 1919. Suite à la mort de l'empereur de Corée déchu par les Japonais, les Coréens manifestent en masse aux cris de «Vive l'indépendance! (Tongnip manse 獨立萬歲).

19. La Reimeikai 黎明会 (《Société de l'Aube») réunit entre 1918 et 1920 des universitaires, libéraux ou socialistes modérés. Les objectifs de cette association sont de lutter contre l'intransigeance et l'intolérance des milieux gouvernementaux. Par le biais de conférences publiques et de brochures, la Reimeikai est de tous les combats pour le suffrage universel, contre les lois répressives, contre la politique coloniale brutale du Japon.

20. Taishō shisō shū 大正思想集 (La Pensée de l'époque Taishō : đEuvres choisies), tome II, édition mise au point par Kano Masanao 鹿野政直, tome 34 de la série Kindai Nihon shisō taikei, op. cit., 1978, postface de Kano Masanao, p. 444. Fukuda Tokuzō était professeur d'histoire économique. Il campait sur des positions libérales proches du courant socialiste modéré.

21. Yoshino Sakuzō, «Pekin gakuseidan no kōdō o manba suru nakare » 北京学生団の行動を漫 黑する勿れ (Ne pas dénigrer le mouvement des étudiants de Pékin), juin 1919, dans Yoshino Sakuzō shū, op. cit., p. 322.

22. Des étudiants, disciples de Yoshino, fondent, en 1918, une association, la Shinjinkai 新人会 (Société des gens nouveaux), qui jouera à l'Université de Tōkyō dans les dix années suivantes, un rôle central dans le mouvement étudiant démocratique. Une association du même type est créée à l'Université Waseda. Une autre existait déjà à l'Université de Kyōto où les étudiants se réunissaient pour discuter de la question sociale en invitant des ouvriers. Les statuts de la Shinjinkai évoquent «la libération de l'humanité » et la "réforme du Japon ». Ces associations constituent les prodromes des "mouvements étudiants ", puissants dès cette époque jusqu'au milieu des années 1970 (exception faite de la période de guerre).

23. Taishō shisō shū II, ibid., p. 444.

24. Comme le fait remarquer Michel Vié dans son ouvrage, c'est moins que la répression pratiquée par la France à Sétif et Guelma en 1945 (entre huit mille et quarante-cinq mille personnes massacrées). En mars 1947, la répression de l'insurrection malgache par les troupes françaises aurait fait quatre-vingts mille morts, chiffre dont on ne peut qu'espérer qu'il soit exagéré. D'autres chiffres évoquent entre cinq et dix mille morts. Contrairement à une légende tenace, le colonialisme japonais n'a pas été plus brutal que les autres. Voir notamment Michel Vié, Le Japon et le monde au XXe siècle, Masson, 1995, 303 p.

25. «Suigen gyakusatsu jiken» 水原虐殺事件 (massacre de Suwŏn), Chūo kōron (juillet 1919), dans Yoshino Sakuzō senshū, tome 9, op.cit. p. 67. La gendarmerie japonaise aurait massacré une trentaine de villageois à Chemuri, dans le canton de Suwŏn.

26. Paull Hobom Shin, The Korean Colony in Chientao, A Study of Japanese Imperialism and Militant Korean Nationalism, 1905-1932, Thèse de doctorat, Université de Washington, 1980, 460 p. 
27. Voir, notamment, la contribution de Yoshino publiée dans le numéro de février 1921 de Chūō kōron, dans Yoshino Sakuzō senshū, tome 9, op. cit., p. 171.

28. «Chōsen seinenkai mondai - Chōsen tōjisaku no kakusei o unagasu » 朝鮮青年会問題一朝鮮 統治策の覚醒を促す (La question des associations de jeunesse en Corée - Ouvrir les yeux sur la politique menée en Corée), Shinjin, 1920, 2-3, dans Yoshino Sakuzō shū, op. cit., p. 298.

29. “Chōsenjin ni omou», Yanagi Sōetsu shū 柳宗悦集 (Æuvres de Yanagi Sōetsu), Tsurumi Shunsuke (éd.), Kindai Nihon shisō taikei, op. cit., t. 24, 1975, 444 p., p. 183.

30. Ibid., p. 178.

31. Ibid., p. 182-183.

32. Sur Yanagi Muneyoshi alias Sōetsu, voir le dossier spécial qui lui est consacré dans le $\mathrm{n}^{\circ} 16$ de Cipango - Cahiers d'études japonaises, 2009. Voir également Sōetsu Yanagi, Artisan et inconnu, la Beauté dans l'esthétique japonaise, adapté par Bernard Leach, L'Asiathèque, 1992, 165 p.

33. Sur la formation du complexe de supériorité des Japonais vis-à-vis des Coréens, voir Lionel Babicz, Le Japon face à la Corée à l'époque Meiji. op. cit.

34. Bernard Leach, Livre du potier, Dessain et Tolra, 1973, $381 \mathrm{p}$.

35. Dans «Chōsen no bijutsu » (L'art coréen), Yanagi parle de « beauté amère » (hishū no bi 悲愁 の美). Voir Yanagi Sōetsu shā, op.cit., p. 223. Plus loin, p. 226, il évoque une «beauté mélancolique » (hiai no bi 悲哀の美).

36. « Chōsenjin ni omou », op. cit., p. 177.

37. Ibid., p. 180-181.

38. Ibid., p. 183.

39. Ibid., p. 181-182.

40. «Chōsen no tomo ni okuru sho» 朝鮮の友に贈る書 (Écrit pour des amis coréens), publié dans Kaizō 改造 en juin 1920, repris dans Yanagi Sōetsu shū, op. cit., p. 185.

41. Ibid., p. 188.

42. «Kare no chōsenyuki » 彼の朝鮮行 (Lui qui se rend en Corée), dans Yanagi Sōetsu shū, op. cit., p. 198.

43. «Chōsenjin ni omou », Yanagi Sōetsu shū, op. cit., p. 179, p. 186.

44. Ibid., p. 181.

45. Ibid., p. 183.

46. Tsurumi Shunsuke, postface, dans Yanagi Sōetsu shū, op. cit., p. 434.

47. «Ushinaharentosuru Chōsen kenchiku no tame ni » 失はれんとする朝鮮建築のために (Pour un monument d'architecture coréenne qu'on va perdre), dans Yanagi Sōetsu shū , op. cit., p. 234.

48. Ibid., p. 236.

49. Ibid., p. 235.

50. Ibid., p. 236. La porte avait été détruite lors des invasions japonaises à la fin du $\mathrm{XVI}^{\mathrm{e}}$ siècle, et restaurée dans les années 1860.

51. Cette porte sera restaurée sur son site originel en 1968 tandis que le bâtiment japonais de style moderne est détruit en 1995 dans le cadre des commémorations du 50e anniversaire de l'indépendance de la Corée.

52. Tsurumi Shunsuke, postface, dans Yanagi Sōetsu shū, op. cit., p. 434.

53. Asakawa Noritaka 浅川伯教 (1884-1964) était instituteur à Séoul tandis que Takumi 浅川巧 (1891-1931), son jeune frère venu le rejoindre en Corée, travaillait comme fonctionnaire de l'administration coloniale des forêts. Takumi avait appris le coréen qu'il parlait couramment. Yanagi Sōetsu séjourne à dix reprises en Corée entre 1914 et 1924. Voir Asahi hyakka Nihon no rekishi 朝日百科日本の歴史 (Encyclopédie Asahi - Histoire du Japon), 1987, fascicule 115, p. 147.

54. Kim Brandt, "Objects of Desire: Japanese Collectors and Colonial Korea", Positions, 8-3, Winter 2000, University of Duke, p. 711-746, p. 730. 
55. Voir Yoshino Sakuzō, «L'affaire du massacre des Coréens », Ebisu, numéro spécial, Le Japon des séismes, n²1, 1999. Texte dans Chūō kōron nov. 1923, publié dans Yoshino Sakuzō senshū, op. cit., tome 9, p. 199-204. On parle de 2613 Coréens assassinés (deux cent trente et un selon la police) et d'environ 170 Chinois (trois selon la police). Selon l'historien Imai Seiichi (1924-), la vérité est sans doute encore au-dessus de ces chiffres pourtant considérables. Voir Imai Seiichi 今井清一, Taishō demokurashii 大正 デモクラシー (La démocratie de Taishō), tome 23 de Nihon no rekishi 日 本の歴史 (Histoire du Japon), Chūkō bunko 中公文庫, 1966, 1971, 585 p., p. 218.

56. Taishō shisō shū, tome II, op. cit. p. 290.

57. Yamakawa Kikue 山川菊栄, Jinshu teki henken, seiteki henken, kaikyū teki henken 人種的偏見、 性的偏見，階級的偏見 (Préjugés de race, préjugés de sexe, préjugés de classe), juin 1924, repris dans Yamakawa Kikue josei kaihō ronshū 山川菊栄女性解放論集 (Essais choisis sur la libération des femmes de Yamakawa Kikue) Iwanami shoten, 1994, 367 p., p. 74-75. Rappelons qu'en septembre 1923, au lendemain du séisme, l'anarchiste Itō Noe 伊藤野枝 qui était avec Yamakawa Kikue l'une des fondatrices de la Sekirankai 赤欄会 (Société des Vagues rouges, 1921-1923) avait été étranglée dans un commissariat de Tōkyō avec son compagnon, le célèbre anarchiste Ōsugi Sakae 大杉栄 (1885-1923) et son jeune neveu.

58. Vera Mackie, Feminism in Modern Japan, Cambridge University press, New York, 2003, 308 p., fait remarquer p. 88 que Yamakawa Kikue fait une différence entre la société japonaise qui apparaît comme une société "genrée ", et les sociétés colonisées, qu'elle considère comme un bloc, sans classes ni genres. Lapsus d'une militante issue du pays colonisateur?

59. Ishibashi Tanzan est bien le seul Premier ministre japonais dont les œuvres complètes font quinze volumes! (publiées en 1970-72 aux éditions Tōyō keizai shinpōsha 東洋経済新報者).

60. Comme le remarque Karatani Kōjin 柄谷行人 dans l'ouvrage de Richard F. Calichman (dir.), Overcoming Modernity, Columbia University Press, New York, 2008, 248 p., p. 108.

61. Voir la postface de Matsuo Takayoshi 松尾尊兌 (dir.), Ishibashi Tanzan hyōronshū 石橋湛山評 論集 (๕uvres critiques d'Ishibashi Tanzan), Iwanami bunko, 1984, 2008, 313 p., p. 293-295.

62. Dès la fin du $x x^{e}$ siècle et les premières années $d u x^{e}$ siècle, Nakae Chōmin 中江兆民 (1847-1901), Ueki Emori, Abe Isoo 安部僟雄 (1865-1949) ou encore le nationaliste nipponiste Miyake Setsurei s'étaient fait, chacun à sa manière, les avocats de la cause d'un Petit Japon, sans pour autant parvenir à créer des relais politiques très structurés.

63. Miura Tetsutarō, «Dai Nipponshugi ka Shō Nipponshugi ka»大日本主義乎小日本主義 乎 (Grand ou Petit Japon ?), 15 avril 1913, repris dans Taishō shisōshū, tome I, édition mise au point pas Imai Seiichi, tome 33 de la série Kindai Nihon shisō taikei, 1978, 507 p., p. 66.

64. Ibid., p. 67.

65. «Shintō wa danjite ryōyū su bekarazu» 青島は断じて領有すべからず (Surtout ne pas s'emparer de Qingdao), éditorial du 15 novembre 1914, in Ishibashi Tanzan hyōronshū, op. cit., p. 52. 66. «Kakon o nokosu gaikō seisaku » 禍根を残す外交政策 (Une politique étrangère qui laisse de côté le problème de fond), éditorial du 5 mai 1915, dans Ishibashi Tanzan hyōronshū, op. cit., p. 56-57.

67. Cité par Kano Masanao, Kindai kokka o kōsōshita shisōkatachi 近代国家を構想した思想家たち (Les penseurs qui ont élaboré l'État moderne), Iwanami junia shinsho 岩波ジュニア新書, 181 p., p. 133.

68. «Senjin bōdō ni taisuru rikai» 鮮人暴動に対する理解 (Comprendre la violence des Coréens), éditorial du 15 mai 1919, dans Ishibashi Tanzan hyōronshū, op. cit. p. 87-88.

69. «Issai o sutsuruno kakugo. Taiheiyōkaigi ni taisuru waga taido»一切を棄つるの覚悟太平洋 会議に対する我が態度 (Soyons prêts à tout abandonner. Notre position sur la conférence du Pacifique), éditorial du 22 juillet 1922, réédité dans Ishibashi Tanzan hyōronshū, op. cit., p. 98-99.

70. Depuis 1889, l'appellation officielle du Japon est «Empire du Grand Japon» (Dai Nippon teikoku). Le royaume coréen devenu à son tour «empire " en 1897 cède à la même mode en 
s'intitulant «Empire de la Grande Corée » (Tae Han Cheguk). À peu près à la même époque, certains évoquaient la « plus grande France ».

71. «Dai Nihonshugi no gensō» 大日本主義の幻想 (Les illusions de la doctrine du Grand Japon), éditorial du 30 juillet 1922, réédité dans Ishibashi Tanzan hyōronshū, op. cit., p. 101.

72. Ibid., p. 113.

73. Ibid., p. 120.

74. Ibid., p. 121.

75. «Manmō mondai kaiketsu no konpon hōshin ikan » 満蒙問題解決の根本方針如何 (Quelles orientations fondamentales pour résoudre la question de la Mandchourie et de la Mongolie?), 26 septembre 1931, Ishibashi Tanzan hyōronshū, op. cit. p. 178.

76. Ibid., p. 180.

77. Voir Saburō Ienaga, The Pacific War, 1931-1945, Pantheon Books, New York, 1978, 336 p., p. 120.

78. Kano Masanao, Kindai Nihon shisō annai 近代日本思想案内 (Guide de la pensée japonaise moderne), Iwanami bunko, 1999, 393 p., p. 290-291. Sur Takeuchi Yoshimi et son rôle ambigu en Chine pendant la guerre, voir Samuel Guex, Entre nonchalance et désespoir, Les intellectuels japonais sinologues face à la guerre (1930-1950), Berne, Peter Lang, 2006, $300 \mathrm{p}$.

79. Ishibashi Tanzan, « Kinrai no sesō tada kotonarazu » 近来の世相ただことならず(Les mœurs politiques de ces derniers temps ne seront pas sans conséquence), éditorial du 18 avril 1931, Ishibashi Tanzan hyōronshū, op. cit., p. 173-174

80. Voir Kurt W. Radtke, "Nationalism and Internationalism in Japan's Economic Liberalism, The Case of Ishibashi Tanzan", in Dick Stegewerns (dir.), Nationalism and Internationalism in Imperial Japan, Routledge, Londres, 2003, 272 p., p. 177.

81. Cité par Matsuo Takayoshi dans Ishibashi Tanzan hyōronshū, op. cit., p. 308.

82. Logique par rapport à lui-même puisqu'il avait été l'un des premiers à demander la reconnaissance par Tōkyō du régime "extrémiste" (bolchévik) en 1918. " Kagekiha seifu o shōnin seyo»過激派政府を承認せよ (Reconnaissons le gouvernement des extrémistes), 25 juillet 1918, dans Taishō shisōshū tome I, op. cit., p. 386.

83. Le courant - très minoritaire au sein du Parti libéral démocrate - dont Ishibashi Tanzan était le leader, s'oppose, en 1957, au projet de traité de sécurité nippo-américain. Pour lui, le Japon devait respecter une stricte neutralité dans l'affrontement des blocs. Il craignait que l'alliance avec les États-Unis n'entraîne de nouveau le Japon dans une aventure militaire.

84. Tanaka Shūsei 田中秀征, Nihon Riberaru to Ishibashi Tanzan, ima seiji ga hitsuyō toshite iru koto 日 本リベラルと石橋湛山いま政治が必要としていること (Le Japon libéral et Ishibashi Tanzan, ce qui serait nécessaire aujourd'hui dans la vie politique), Kōdansha 講談社, 2004, 242 p.

85. Mark R. Peattie, “Japanese Attitudes Toward Colonialism”, dans Myers and Peattie (dir.), The Japanese Colonial Empire, op. cit., p. 84.

86. Il s'agit sans doute là des premiers enseignements dispensés au Japon consacrés à la "politique coloniale» mais aucune chaire n'avait été alors créée. Yamamoto Miono enseigne la politique coloniale à la Faculté de Droit, en 1912, avant qu'une chaire de professeur ne soit ouverte officiellement, avec la création de la Faculté d'Économie, en 1919, chaire qui lui fut confiée.

87. Chaire d'«études coloniales» (shokumingaku kōza 植民学講座) créée dans la Faculté d'Agronomie. En 1910, Nagai Ryūtarō 永井柳太郎 (1881-1944) est nommé à Waseda sur un poste semblable.

88. Chaire de «politique coloniale» (shokumin seisaku kōza 植民政策講座), créée au sein de la Faculté de Droit puis rattachée, en 1919, à la Faculté d'Économie.

89. Andrew E. Barshay, "Postwar Social and Political Thought, 1945-90", in Bob Tadashi Wakabayashi (dir.), Modern Japanese Thought, Cambridge University Press, 1998, 414 p., p. 298.

90. «Chōsen tōji no hōshin» 朝鮮統治の方針 (Les Orientations de la politique coréenne), Chūō kōron, juin 1926, reprod. Taishō shisō shū, tome II, op. cit., p. 383. 
91. Ibid., p. 384.

92. Ibid., p. 390.

93. Ibid., p. 385.

94. Ibid., p. 392. Le droit de vote fonctionne en ce temps selon une discrimination territoriale et non ethnique. Rappelons que les Japonais résidant en Corée ne votaient pas mais que les Coréens du Japon votaient. Un député coréen a été deux fois élu au parlement impérial dans les années 1930. Quoi qu'il en soit, aucun empire colonial n'a jamais donné le droit de vote aux indigènes.

95. Ibid., p. 391.

96. Ibid., p. 392.

97. « Chōsen tōji no hōshin » dans Taishō shisō shū, vol. II, op. cit., p. 394.

98. Cité par Myers and Peattie, The Japanese Colonial Empire, op. cit., p. 117.

99. Asada Kyōji 浅田橋二, “Yamamoto Miono no shokuminron»山本美越乃の 植民論 (Le discours colonial de Yamamoto Miono), dans Keizaigaku ronshū 経済学論集, Komazawa daigaku 駒 沢大学, vol. 18, $1^{\mathrm{e}}$ partie et $2^{\mathrm{e}}$ partie, novembre-décembre 1986, p. 18 et seq. de la $1^{\mathrm{e}}$ partie.

100. " Chōsen tōji no hōshin », dans Taishō shisō shū II, op. cit.p. 393.

101. Ibid. p. 394.

102. Yanaihara Tadao, Teikokushugi ka no Taiwan, op.cit. Outre l'habituelle simplification des sinogrammes, Wakabayashi Masahiro publie ici des notes explicatives d'une grande utilité. Cette étude sur Taiwan n'est pas la première du genre, puisque Yamamoto Miono avait déjà publié une longue étude : «Taiwan gikai setchi seigan undō to kako no sōtokuseiji » 台湾議会設置請願運動 と過去の総督政治 (Le mouvement de pétition en faveur de la mise en place d'une assemblée à Taiwan et la politique de la Résidence Générale autrefois), in Gaikō jihō 外交時報 (Informations diplomatiques), nº 488, avril 1925, cité par Asada Kyōji, op. cit. Yamakawa Kikue de son côté avait rédigé, elle aussi, une étude sur Taiwan, en 1925. Yamakawa Kikue, Shokumin seisakuka no Taiwan 植民政策下の台湾 (Taiwan sous le régime colonial), 1925, repris dans Yamakawa Kikue zenshū 山 川菊栄全集 (CEuvres complètes de Yamakawa Kikue), tome 7, Keisō shobō 勁草書房, 1966.

103. Kuwabara Takeo 桑原武夫, Nihon no meicho, Kindai no shisō 日本の名著近代の思想 (Auteurs japonais, la pensée moderne) Chūkō shinsho 中公新書, 1962, 310 p., p. 250.

104. Yanaihara Tadao, Teikokushugi ka no Taiwan, op. cit., postface de Wakabashi Masahiro, p. 339. L'ouvrage est de nos jours régulièrement republié à Taiwan.

105. Avant l'arrivée des Japonais, la population de l'île de Taiwan est composée, d'une part, de populations locales proches ethniquement des anciens peuples malais et parlant des langues d'origine austronésienne et, d'autre part, de colons chinois pour la plupart provenant de la province continentale voisine du Fujian, arrivés plus récemment depuis le XVII ${ }^{\mathrm{e}}$ siècle. En fait, à l'époque des Qing, ces populations sont désignées sous le nom de Fan 番 (Ban en japonais), eux mêmes divisés en Fan «civilisés" (shufan/jukuban 塾番), qui habitent les plaines, et Fan «sauvages» (shengfan/seiban 生番) qui vivent dans les montagnes. Les Fan «civilisés » sont en fait souvent des métis, l'immigration en provenance du Fujian étant essentiellement masculine. Les Japonais reprennent le terme de Fan/Ban en transformant l'idéogramme en 蕃 (utilisé en chinois pour designer les barbares), et prennent l'habitude de désigner les populations les plus anciennes de l'île sous des expressions à connotation péjorative, doban (tufan) 土蕃, doi (tuyi) 土 夷 ou dohi (tufei) 土匪 (indigènes sauvages). En 1935, à l'occasion de la grande exposition de Taiwan, ils modifient ces appellations, parlant désormais de Heiho-zoku (pingbu-zu) 平埔族 (anciennes populations pacifiées et très métissés) et de Takasago-zoku (Gaosha-zu) 高砂族 (peuples dont la pacification avait été durement acquise mais qui seront utilisés comme volontaires dans l'armée japonaise après 1942). Ces derniers, qui représentent aujourd'hui $2 \%$ environ de la population de l'île, sont de nos jours plutôt connus sous le nom de minorités Gaoshan-zu 高山族. Voir note de Wakabayashi Masahiro, in Yanaihara Tadao, Teikokushugi ka no Taiwan, op. cit., p. 28. Yanaihara montre comment dès l'époque des Qing, les Fan (Ban) devenus 
minoritaires ont été spoliés de leurs terres par les immigrants chinois, c'est-à-dire comment Taiwan a toujours été placée sous régime colonial depuis le XVII ${ }^{\mathrm{e}}$ siècle (ibid., p. 37-40).

106. Yanaihara Tadao, ibid., p. 26.

107. Ibid., p. 25.

108. Ibid., p. 23.

109. Ibid., p. 245.

110. Ibid., p. 316.

111. Kuwabara Takeo, Nihon no meicho, Kindai no shisō, op. cit., écrit p. 246 que, vers 1929, quand il était collégien en province, un "indigène " de Taiwan était venu dans son école. Vêtu d'une tenue bariolée, bronzé, il avait effectué devant les élèves une quelconque performance artistique. Le jeune Kuwabara avait été frappé, à l'époque, dit-il, par le côté «spectacle de montreur de foire »- " zoo humain » dirait-on de nos jours -, de la visite, et surtout par l'air désabusé et fatigué de l'indigène en question, par le côté finalement cruel de la performance exigée de cet homme transformé en " panneau publicitaire » de la politique coloniale du Japon à Taiwan. À ce propos, on peut noter que les récentes critiques des exhibitions coloniales sont, semble-t-il, anciennes dans le cas japonais. Voir Nicolas Bancel, Pascal Blanchard, Gilles Boëtsch, Eric Deroo, Sandrine Lemaire, Zoos humains, Au temps des exhibitions humaines, Paris, La Découverte, 486 p., 2004.

112. Ibid., p. 304, et surtout p. 317.

113. Yanaihara Tadao, Teikokushugi ka no Taiwan, op. cit., postface de Wakabashi Masahiro, p. 347.

114. Yanaihara Tadao, Teikokushugi ka no Taiwan, op. cit., postface de Wakabashi Masahiro, p. 351. Malgré l'outrance de son propos, Hosokawa n'a pas tout à fait tort si on considère que la chaire de politique coloniale est supprimée en 1945 avec l'effondrement de l'empire. Après guerre, elle change de dénomination et devient chaire d' " économie internationale » (kokusai keizai ron kōza 国際経済論講座). Quoi qu'il en soit, Yanaihara et Hosokawa furent tous deux emprisonnés pendant la guerre. Hosokawa fut l'auteur d'une histoire coloniale Shokumin shi 植民史, republié dans Hosokawa Karoku chosaku shū 細川嘉六著作集 (Euvres choisies de Hosokawa Karoku), tome II, Rironsha 理論社, 1972, 494 p.

115. En avril 1923, dans le premier numéro de Akahata 赤旗 (Le Drapeau rouge), le journal clandestin du PC japonais, on trouve un court article intitulé « Chōsen kaihō mondai to musan kaikyū » 朝鮮解放問題と無産階級 (La question de la libération de la Corée et le prolétariat), Cf. Taishō shisō shū, tome II, op. cit., p. 444.

116. Yamakawa Hitoshi, Shokumin seisakuka no Taiwan, op. cit.

117. Itō Teruo, Ajia to Kindai Nihon, Hanshinryaku no shisō to undō, op. cit., p. 142-148.

118. «Manshū mondai » 満州問題 (La Question mandchoue), dans Yanaihara Tadao zenshū 矢内原 忠雄全集 (๕uvres complètes de Yanaihara Tadao), tome 2, Iwanami shoten, 1965, 686 p.

119. 《Teikoku shugi kenkyū » 帝国主義研究 (Recherches sur l'impérialisme), in Yanaihara Tadao zenshū, op. cit., tome 4, Iwanami shoten, 1965, 630 p., p. 340.

120. Yanaihara Tadao, "Kirisuto kyō ni okeru heiwa no risō» (L'idéal de la paix dans le christianisme), in Kirisutosha no shinkō 5 Minzoku to heiwa キリスト者の信仰〈5〉民族と平和 (La foi des chrétiens - vol. 5 - Peuples et Paix), Iwanami shoten, 1982, 662 p., p. 117.

121. Narita Ryūichi, Taishō demokurashii, op. cit., p. 151.

122. Ibid., p. 152.

123. Nishikawa Nagao 西川長夫, Shin Shokuminchishugiron Gurobarizêshonka jidai no shokuminchishugi o tou〈新〉植民地主義論 グローバル化時代の植民地主義を問う (Essai sur le néo-colonialisme. Interrogations sur le colonialisme à l'heure de la mondialisation), Heibonsha 平凡社, 2006, 280 p. Nishikawa évoque un colonialisme intériorisé (naimenka sareta shokuminchishugi 内面化された植民地主義), p. 25 et seq.

124. Claude Liauzu, Histoire de l'anticolonialisme en France, Paris, Armand Colin, 2007, 302 p. 


\section{RÉSUMÉS}

Dès les débuts de la mise en place de politiques coloniales, certaines voix se sont élevées au Japon pour critiquer cette nouvelle forme de domination sur des populations d'outre-mer, puis avec les années dites de la démocratie Taishō (1912-1926), cette critique peu connue mais pourtant explicite du colonialisme est devenue plus audible, avant d'être de nouveau progressivement étouffée à la fin des années 1930. Très vite, des doutes naissent, au Japon même, sur la possibilité d'assimiler les peuples coloniaux. On peut distinguer trois courants principaux qui s'en prennent aux politiques coloniales du Japon. D'abord un courant «moral » qui émerge essentiellement comme critique des méthodes répressives policières et militaires dans les colonies, surtout au lendemain des mouvements de 1919 en Corée. Il existe par ailleurs un courant "économiste » produit de la pensée libérale occidentale. Ce courant est anticolonial par principe parce qu'il pense que les colonies coûtent plus chères qu'elles ne rapportent et se préoccupe peu de la situation des peuples colonisés, sinon pour proclamer que les mouvements politiques qui y naissent conduisent inéluctablement à l'indépendance et donc à la défaite programmée du Japon. Enfin, il apparaît une critique plus originale du système au sein même de l'université japonaise parmi certains professeurs chargés d'enseigner précisément les politiques coloniales, qui aboutissent à l'idée que le home rule ou l'indépendance des colonies est inéluctable.

\section{INDEX}

Index géographique : Corée

Mots-clés : Ishibashi Tanzan (1884-1973), politique coloniale, préservation des cultures locales, savoir colonial, Yamakawa Kikue (1890-1980), Yanaihara Tadao (1893-1961), Yanagi Sōetsu

(1889-1961), Yoshino Sakuzō (1878-1933), colonialisme

Thèmes : économie, histoire

Keywords : Korea, Ishibashi Tanzan (1884-1973), Colonial Policy, Preservation of Local Cultures, Colonial Knowledge, Yamakawa Kikue (1890-1980), Yanaihara Tadao (1893-1961), Yanagi Sōetsu (1889-1961), Yoshino Sakuzō (1878-1933), Colonialism, History, Economy, Taishō Period

Index chronologique : Taishō (1912-1923)

\section{AUTEUR}

\section{PIERRE-FRANÇOIS SOUYRI}

Université de Genève 